\title{
Global Dynamics Renders Protein Sites with High Functional Response
}

Yiğit Kutlu, ${ }^{1}$ Nir Ben-Tal ${ }^{2}$ and Turkan Haliloglu ${ }^{*}, 1$

1Department of Chemical Engineering and Polymer Research Center, Bogazici University, Bebek 34342 , Istanbul, Turkey.

${ }^{2}$ Department of Biochemistry and Molecular Biology, George S. Wise Faculty of Life Sciences, Tel Aviv University, Tel Aviv 6997801, Israel.

*E-mail: halilogt@boun.edu.tr 


\section{Supplementary Figures \& Tables}

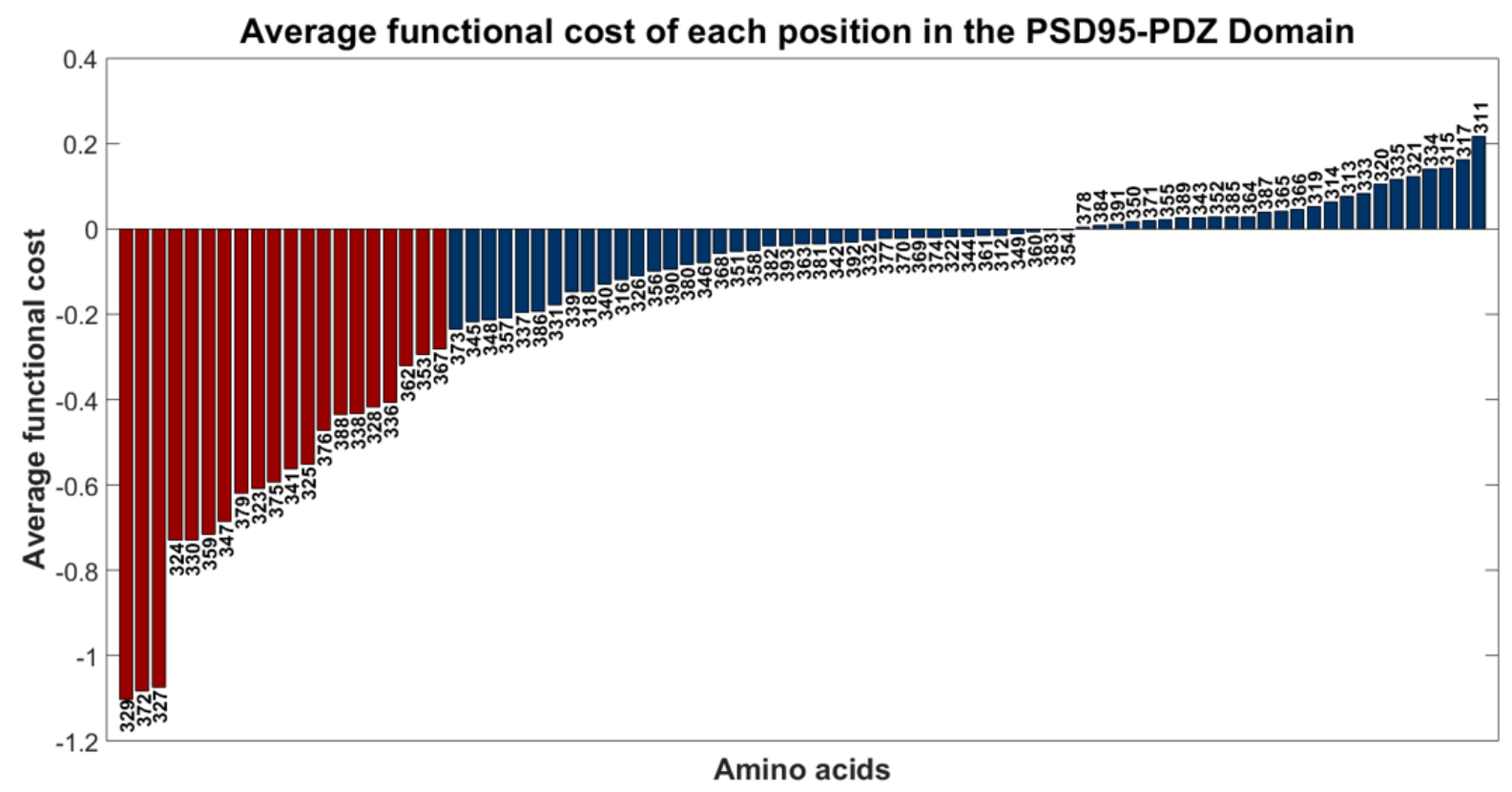

Figure S1. The average functional cost of all the mutations per amino acid position for the PSD95PDZ domain, ranked by severity. Mutation-sensitive positions considered in the dynamic analysis of Figures 1 and S8 are marked in red. The full spectrum is used in Figure S10 and Table S2.

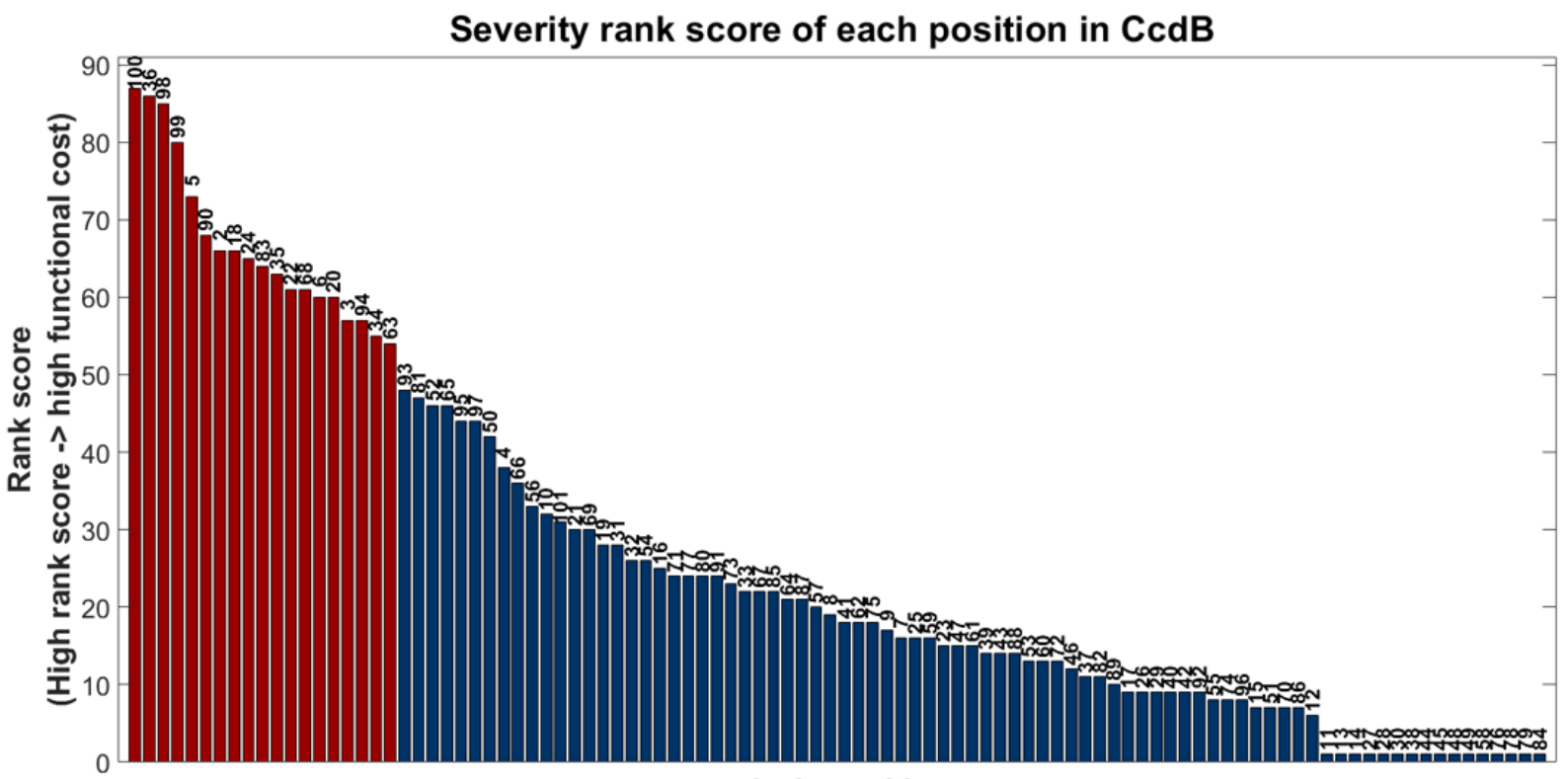

Amino acids

Figure S2. The average functional cost of all the mutations per amino acid position for CcdB, ranked by their Rank Score. High Rank Score means high functional cost (negative fitness effect). Mutation sensitive positions considered in the dynamic analysis are in red. 


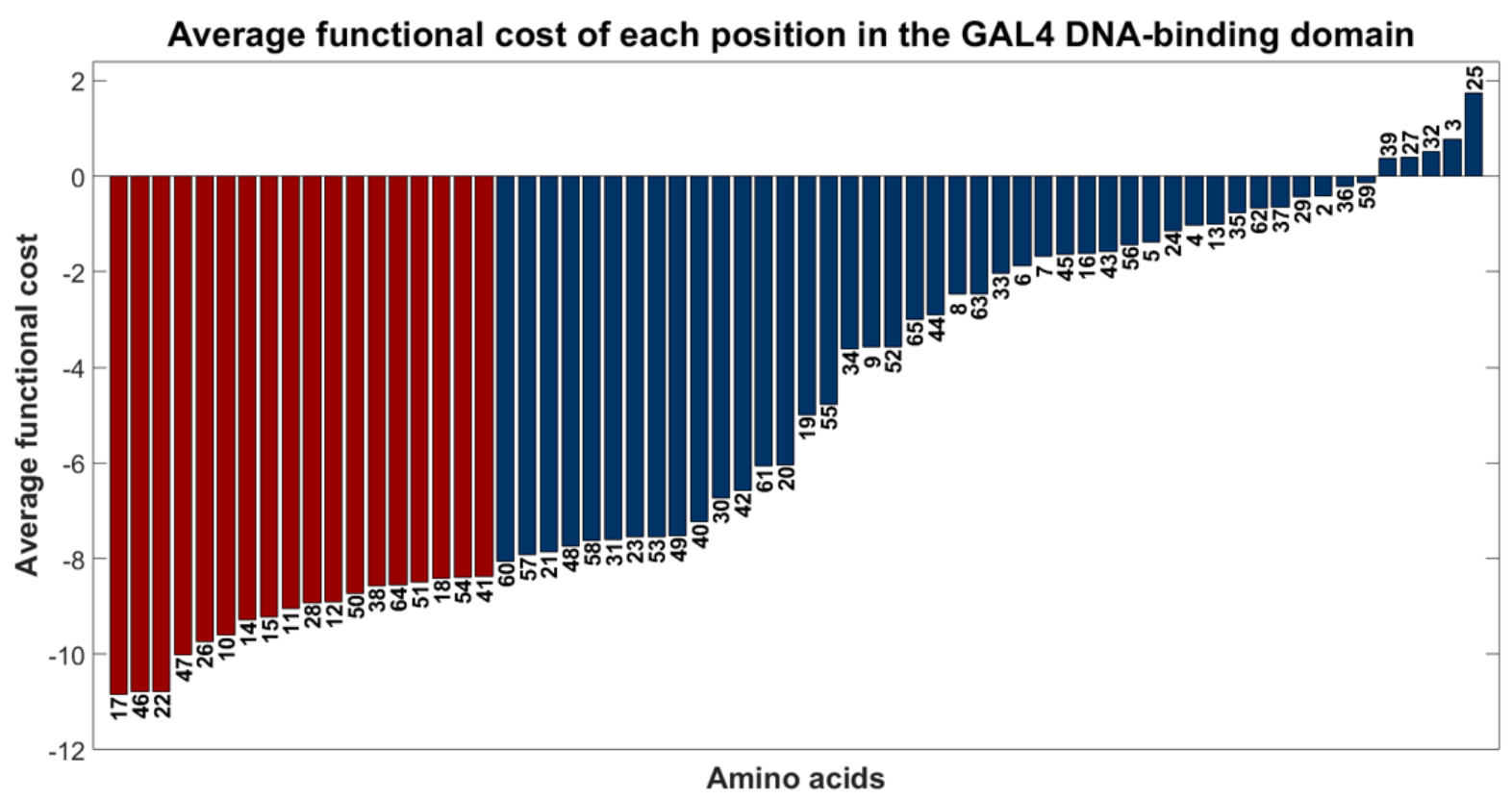

Figure S3. The average functional cost of all the mutations per amino acid position for the GAL4 DNA binding region, ranked by severity. Mutation sensitive positions considered in the dynamic analysis are in red.

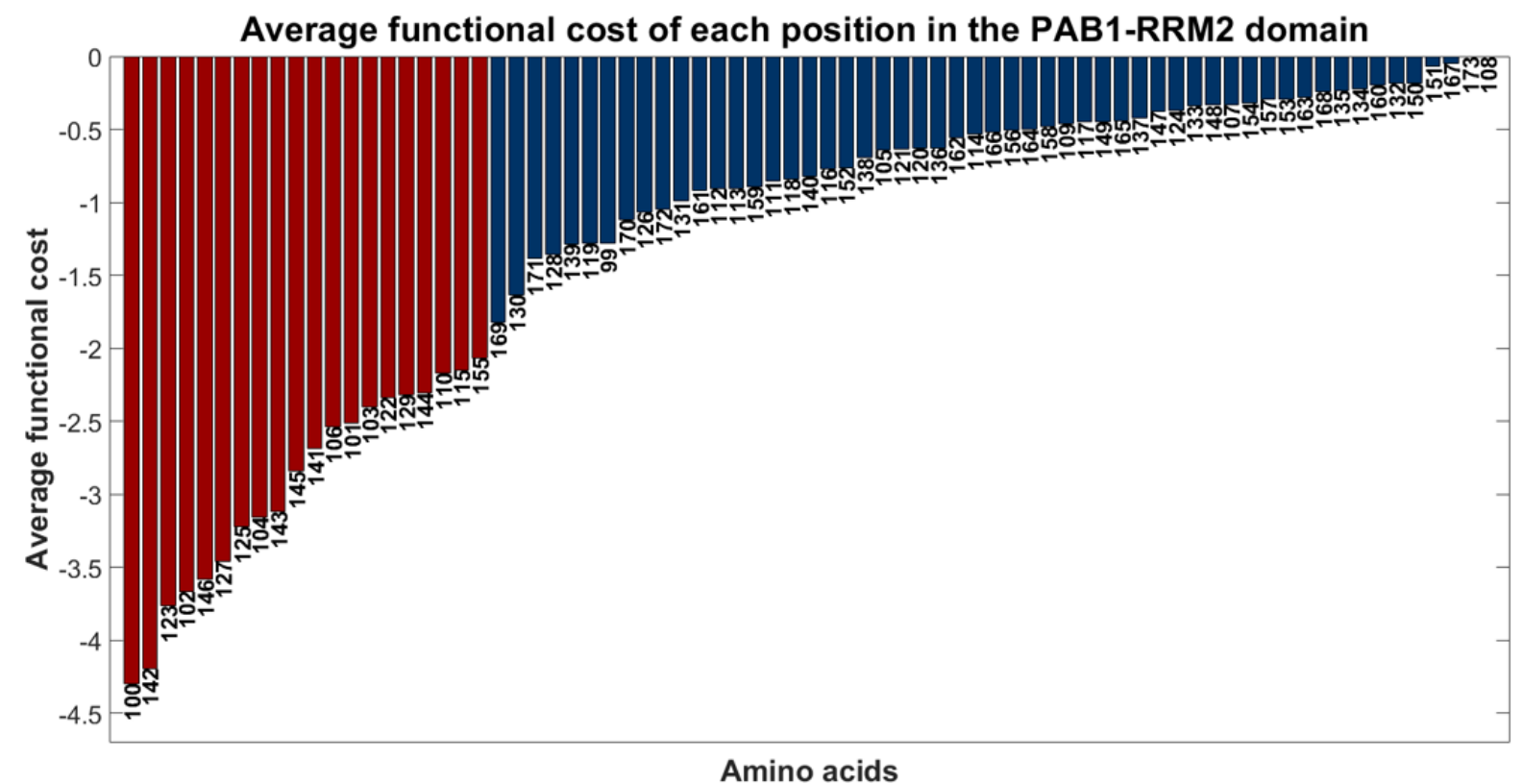

Figure S4. The average functional cost of all the mutations per amino acid position for the PAB1-RRM2 domain, ranked by severity. Mutation sensitive positions considered in the dynamic analysis are in red. 


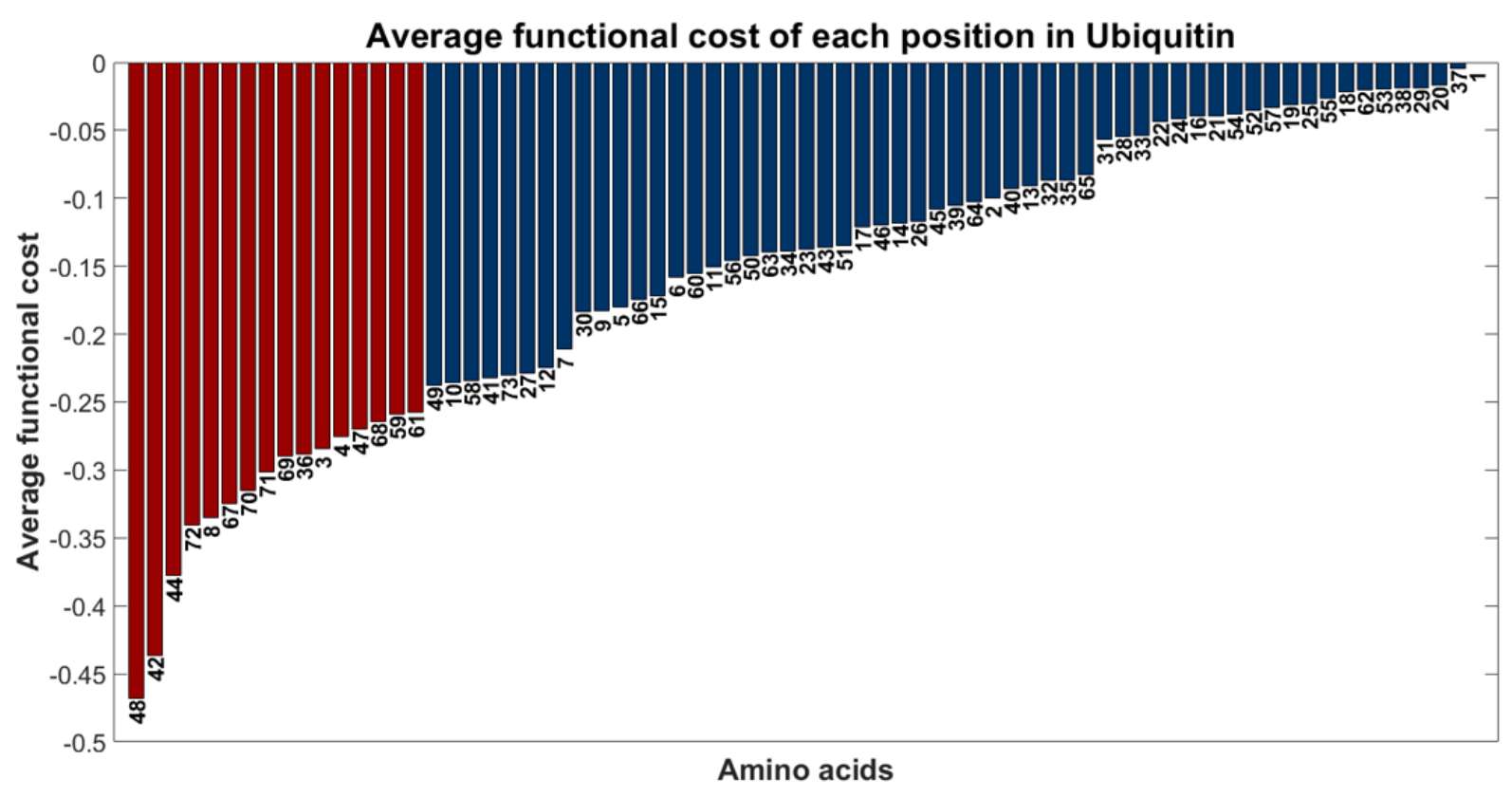

Figure S5. The average functional cost of all the mutations per amino acid position for ubiquitin, ranked by severity. Mutation sensitive positions considered in the dynamic analysis are in red.

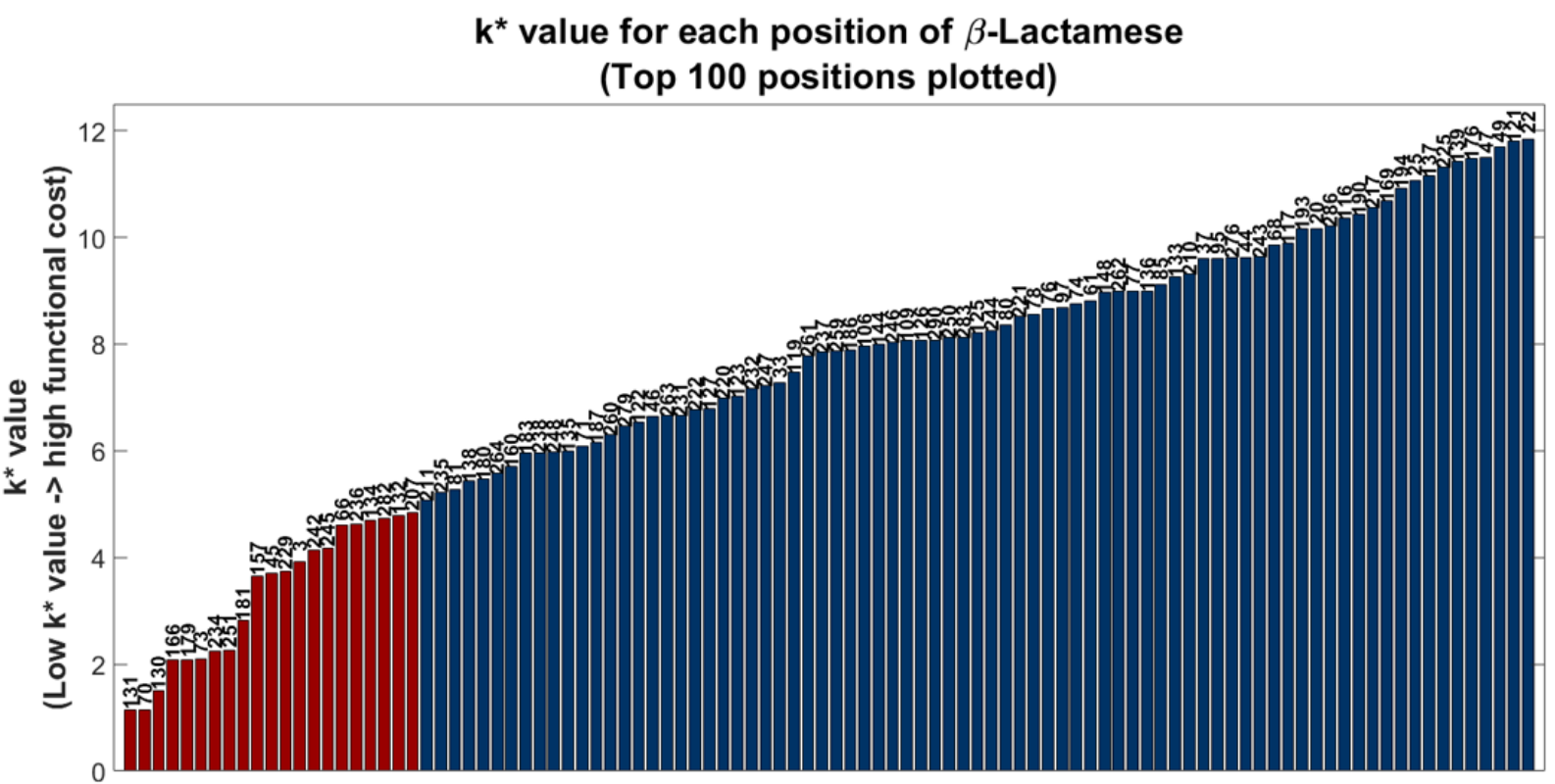

Amino acids

Figure S6. The average functional cost of all the mutations for 100 amino acid positions in $\beta$ lactamase, given as $k^{*}$ score. $k^{*}$ score is inversely proportional to fitness scores of the mutations for each residue. Positions identified as mutation-sensitive are marked in red. 


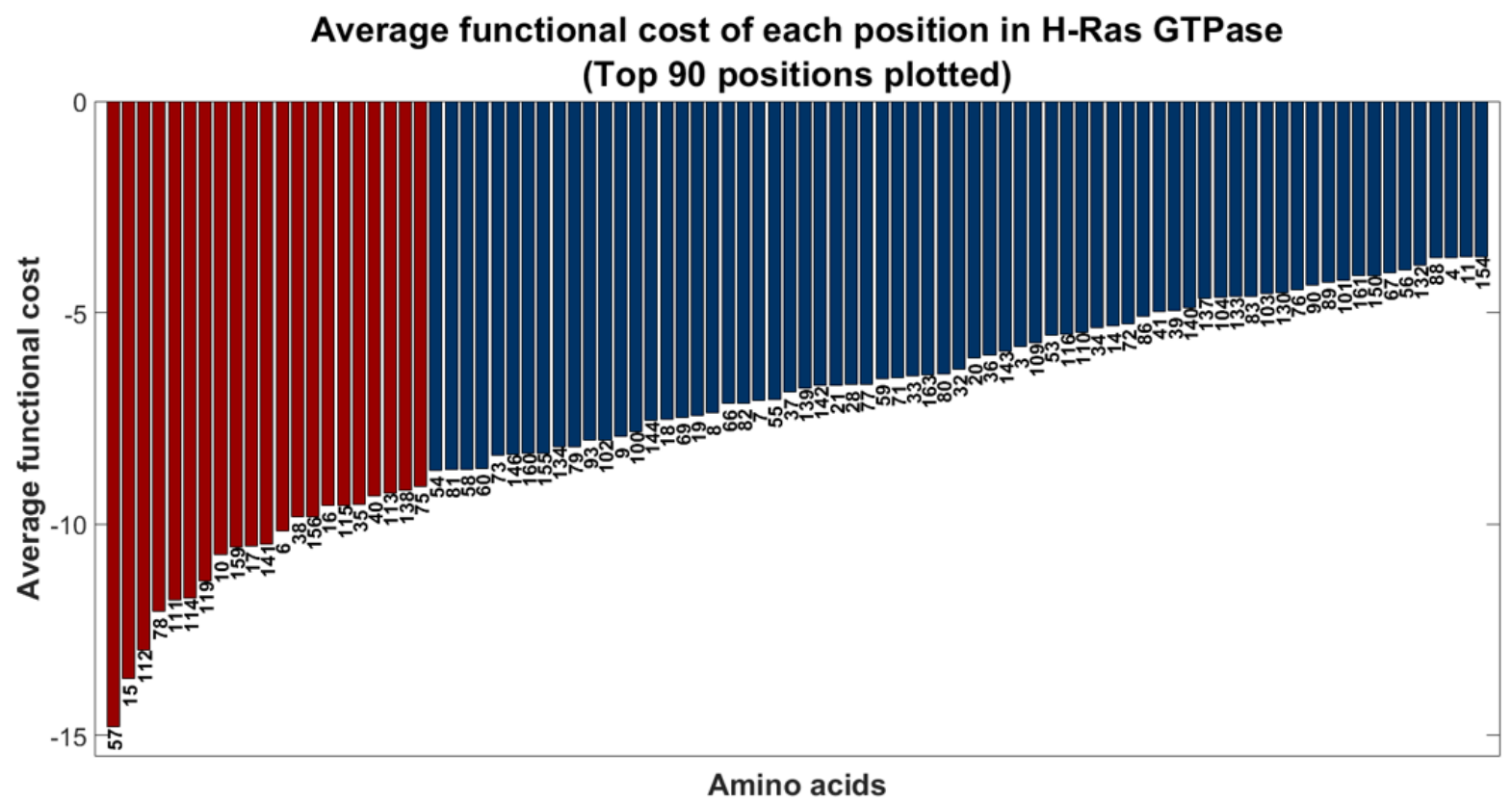

Figure S7. The average functional cost of all the mutations per amino acid in 90 positions for $\mathrm{H}$ Ras GTPase, ranked by severity. Mutation sensitive positions considered in the dynamic analysis are marked in red. 

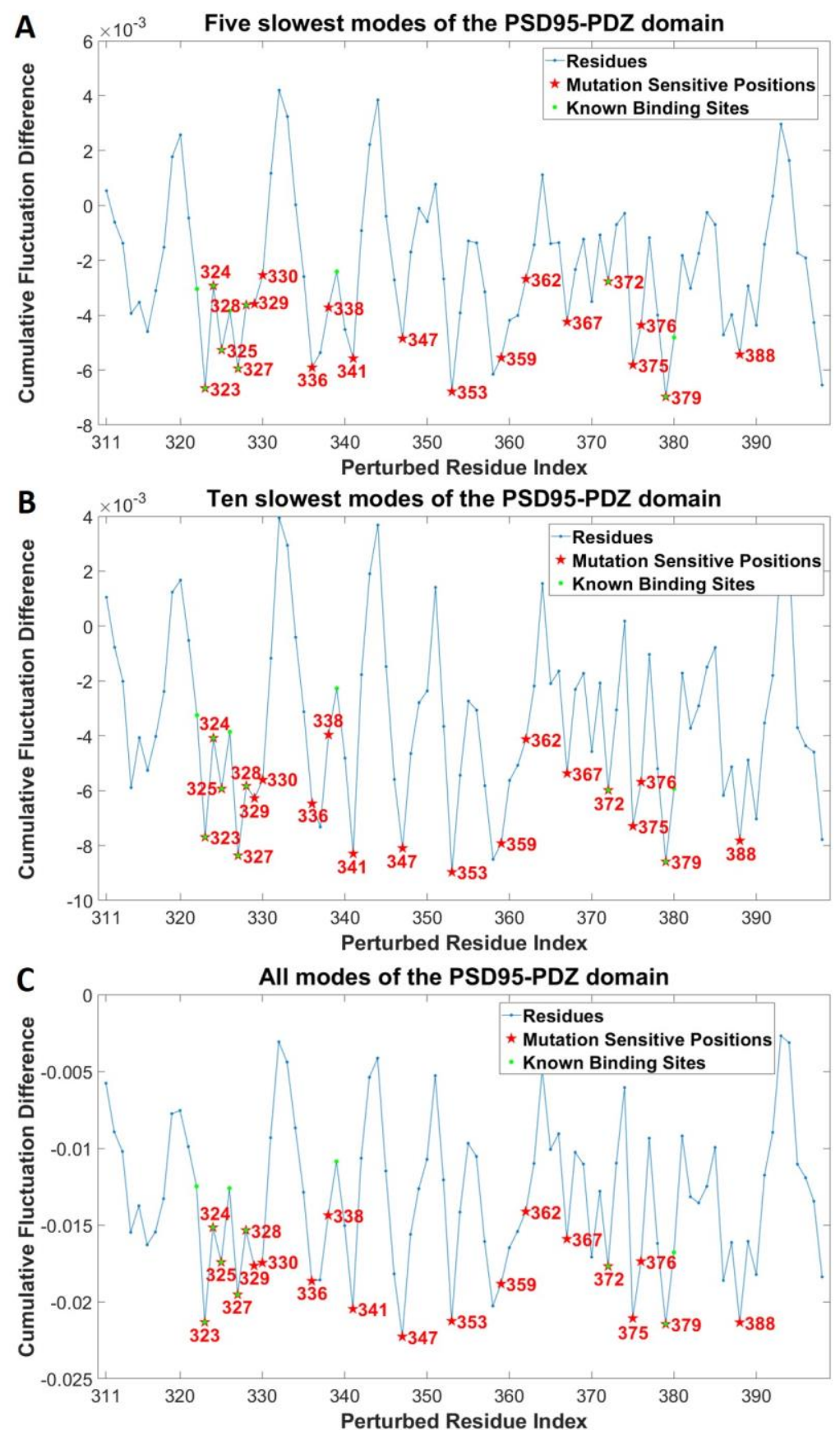

Figure S8. Cumulative residue fluctuations difference profiles of the PSD95-PDZ domain upon straining perturbation on each residue for different mode selections. Mutation sensitive positions, detected using the deep sequencing data, are marked as red asterisks, and binding site residues as green asterisks. (A) The five slowest modes. (B) The ten slowest modes. (C) All modes. Mutation sensitive positions, detected using the deep sequencing data, are marked as red asterisks, and binding site residues as green asterisks. Binding site information is obtained from the crystal structure (PDB ID: 1BE9) entry in PDBsum ${ }^{67}$. 
A Average functional cost and cumulative fluctuation difference in the three slowest modes of the PSD95-PDZ domain

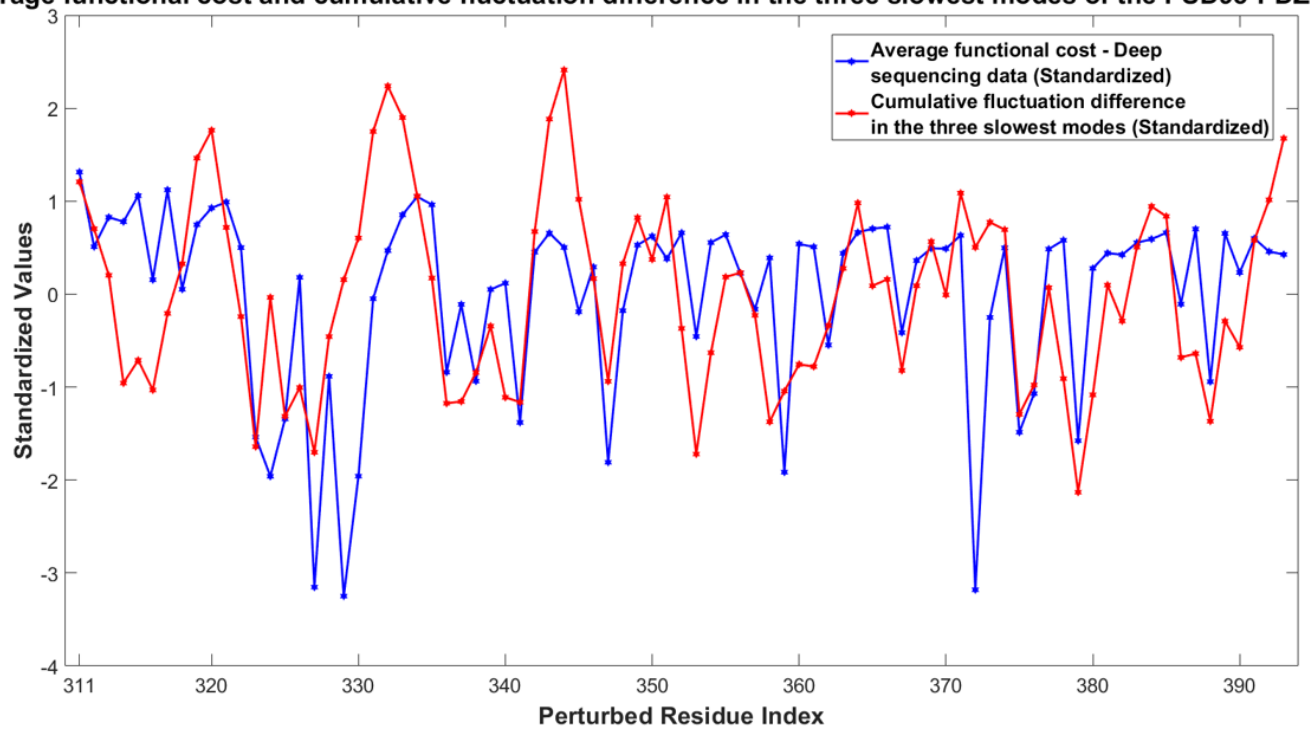

B

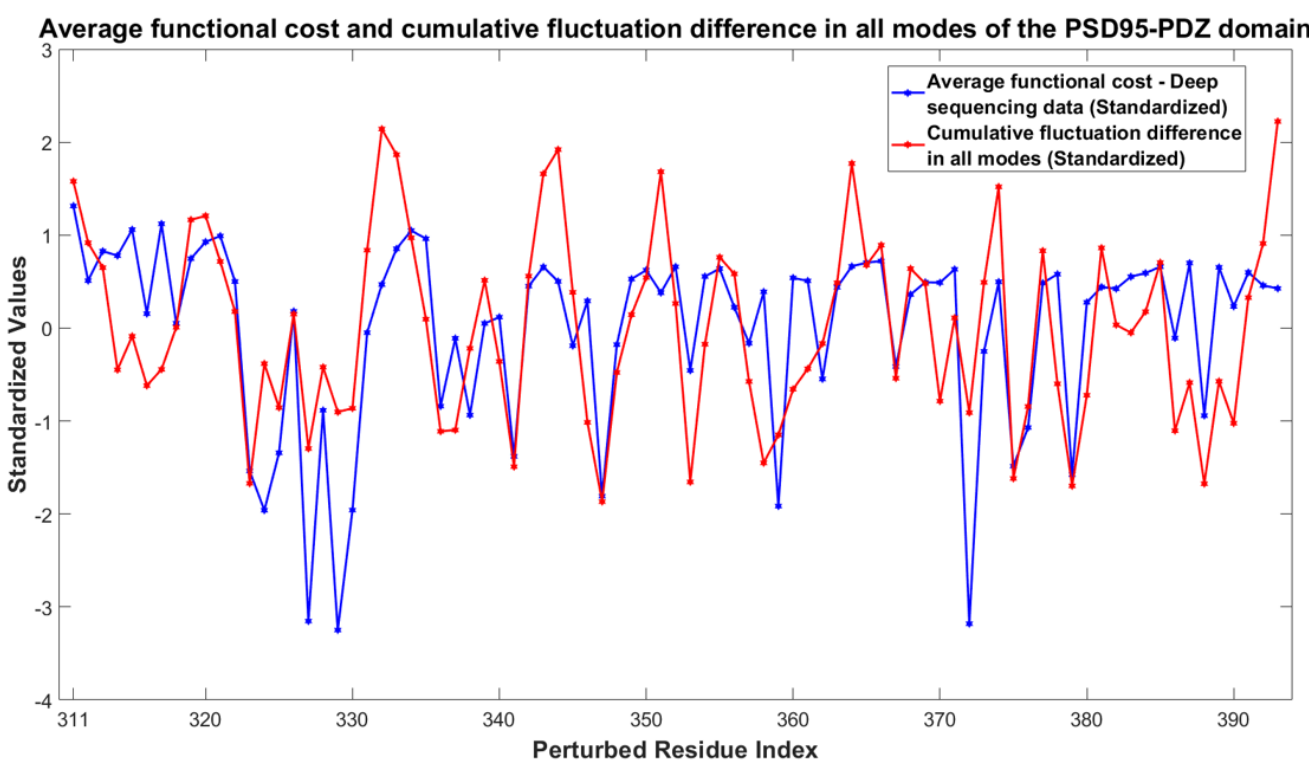

Figure S9. The average functional cost of all the mutations per amino acid position plotted with cumulative residue fluctuations difference profiles of the PSD95-PDZ domain upon straining perturbation on each residue for different mode selections. (A) The three slowest modes. (B) All modes. For comparison, the two data types are standardized 


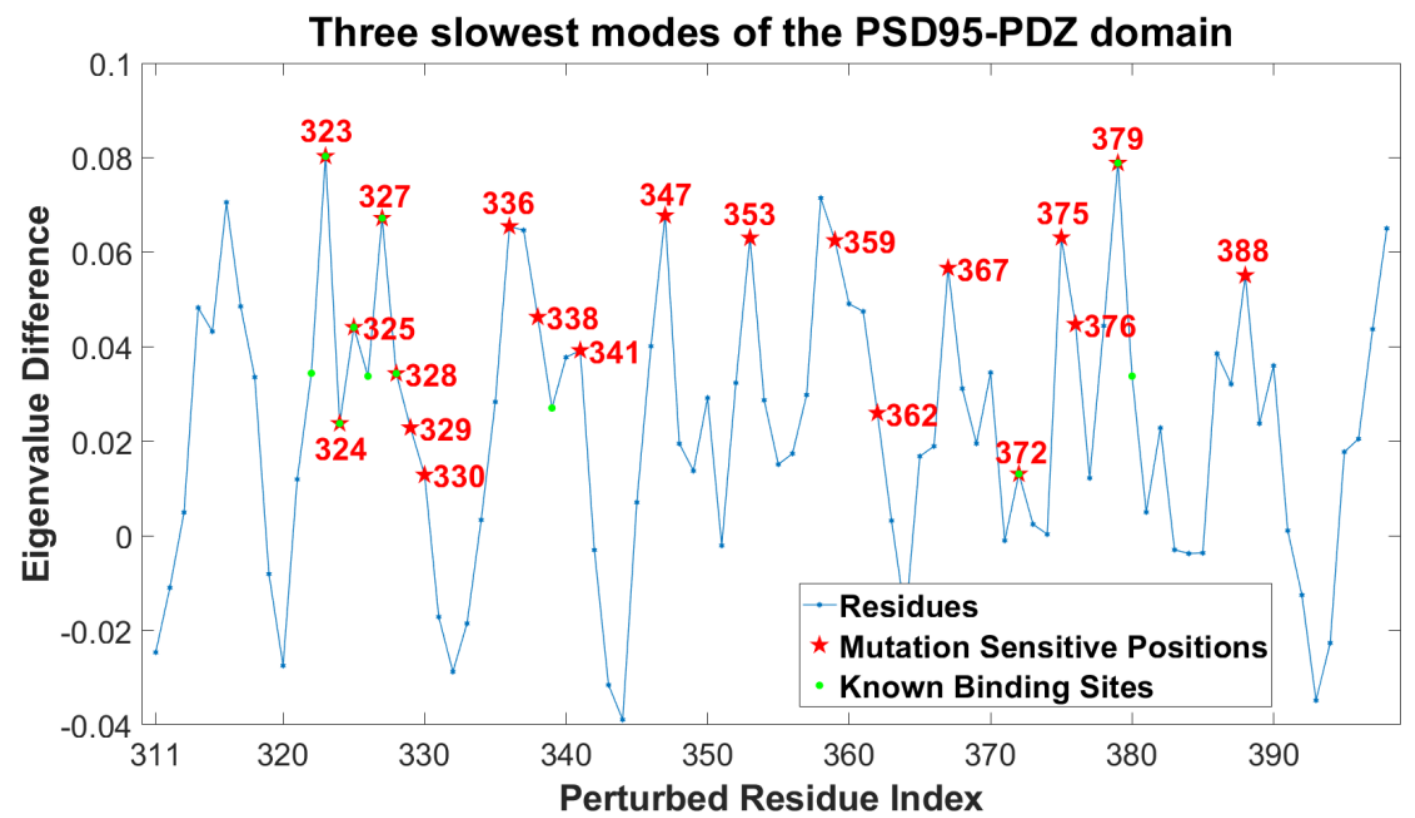

Figure S10. Cumulative eigenvalue difference of the three slowest modes upon strain perturbation of each position in the PSD95-PDZ domain.

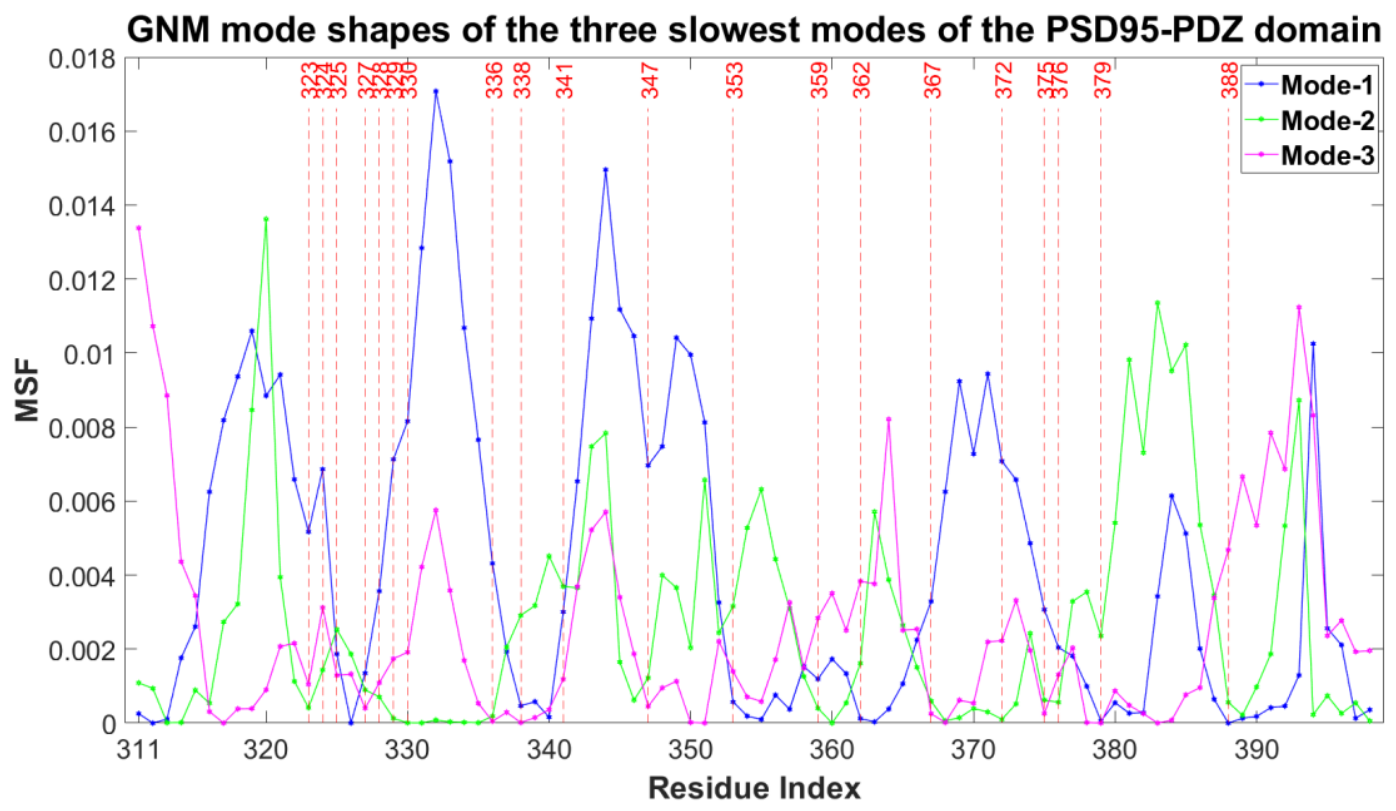

Figure S11. Squared fluctuations of residues in the slowest, second slowest, and third slowest modes of the PSD95-PDZ domain. Mutation sensitive positions, detected using the deep sequencing data, are marked in red dashed lines. 


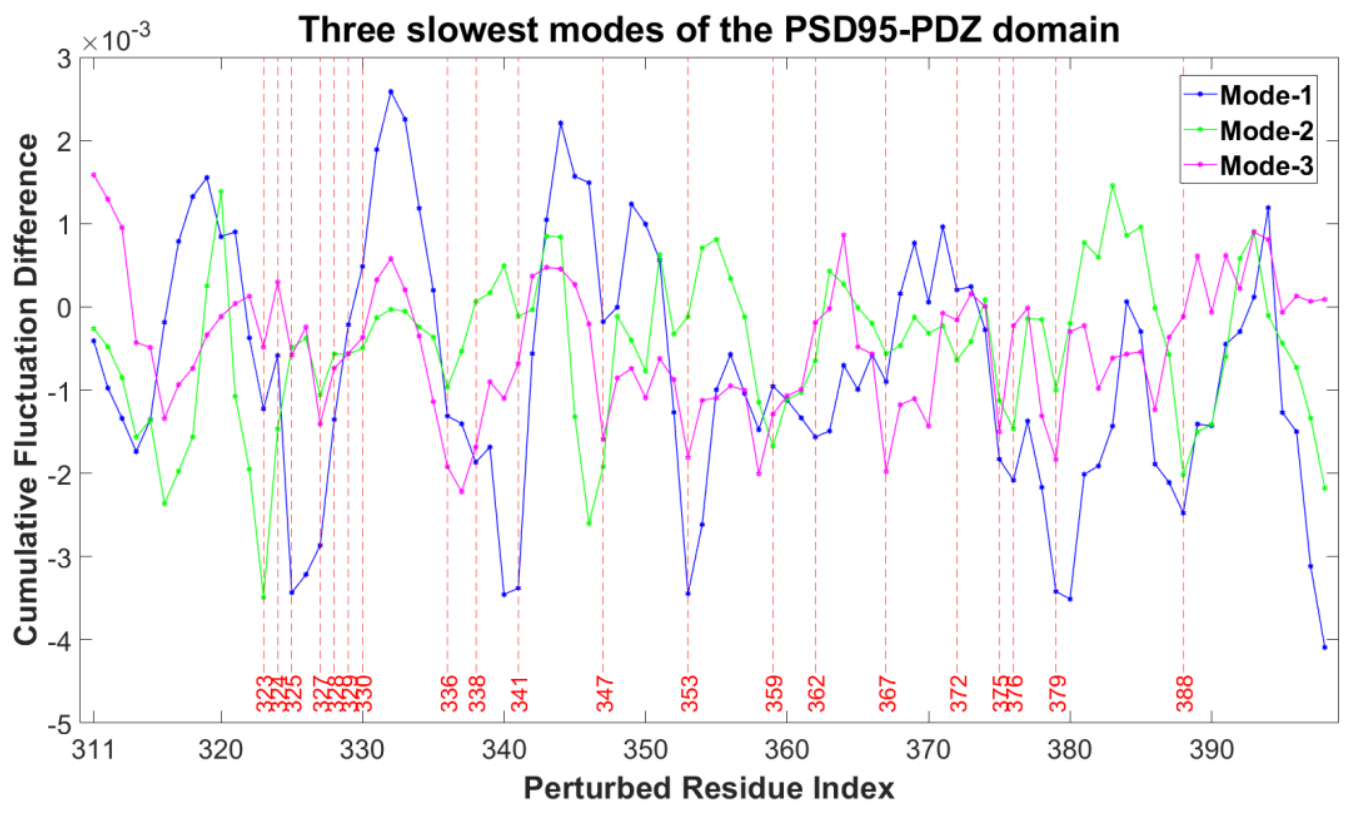

Figure S12. Cumulative residue fluctuation difference profile upon strain perturbation of each position in the PSD95-PDZ domain in the slowest, second slowest, and third slowest modes. Mutation sensitive positions, detected using the deep sequencing data, are marked in red dashed lines. 


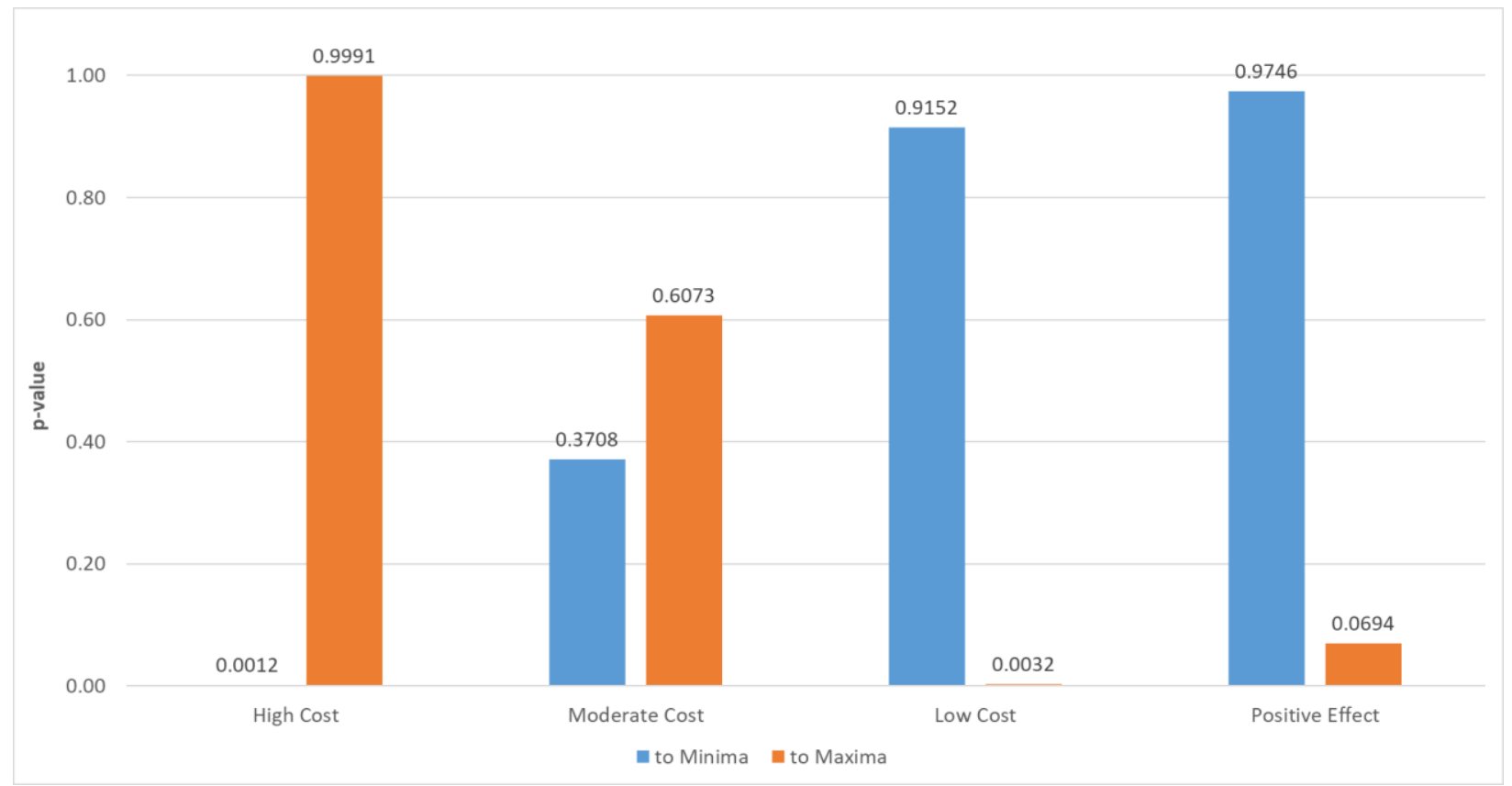

Figure S13. Statistical significance of the correlation between various groups of mutation sensitive positions of the PSD95-PDZ domain, detected in the deep sequencing experiments, and the dynamics perturbation analysis as reflected by the three slowest modes of motion. $p$-values of the correlation between clusters of amino acid positions with different sensitivity to mutations vs. the minima (orange bars) and maxima (blue bars) of the cumulative residue fluctuations profile of the PSD95-PDZ domain. As the functional cost decreases and reaches positive effect on fitness, the correlation with minima positions decreases, whereas in the opposite direction the same behavior is observed towards maxima positions. $p$-value calculations are based on the distances. 

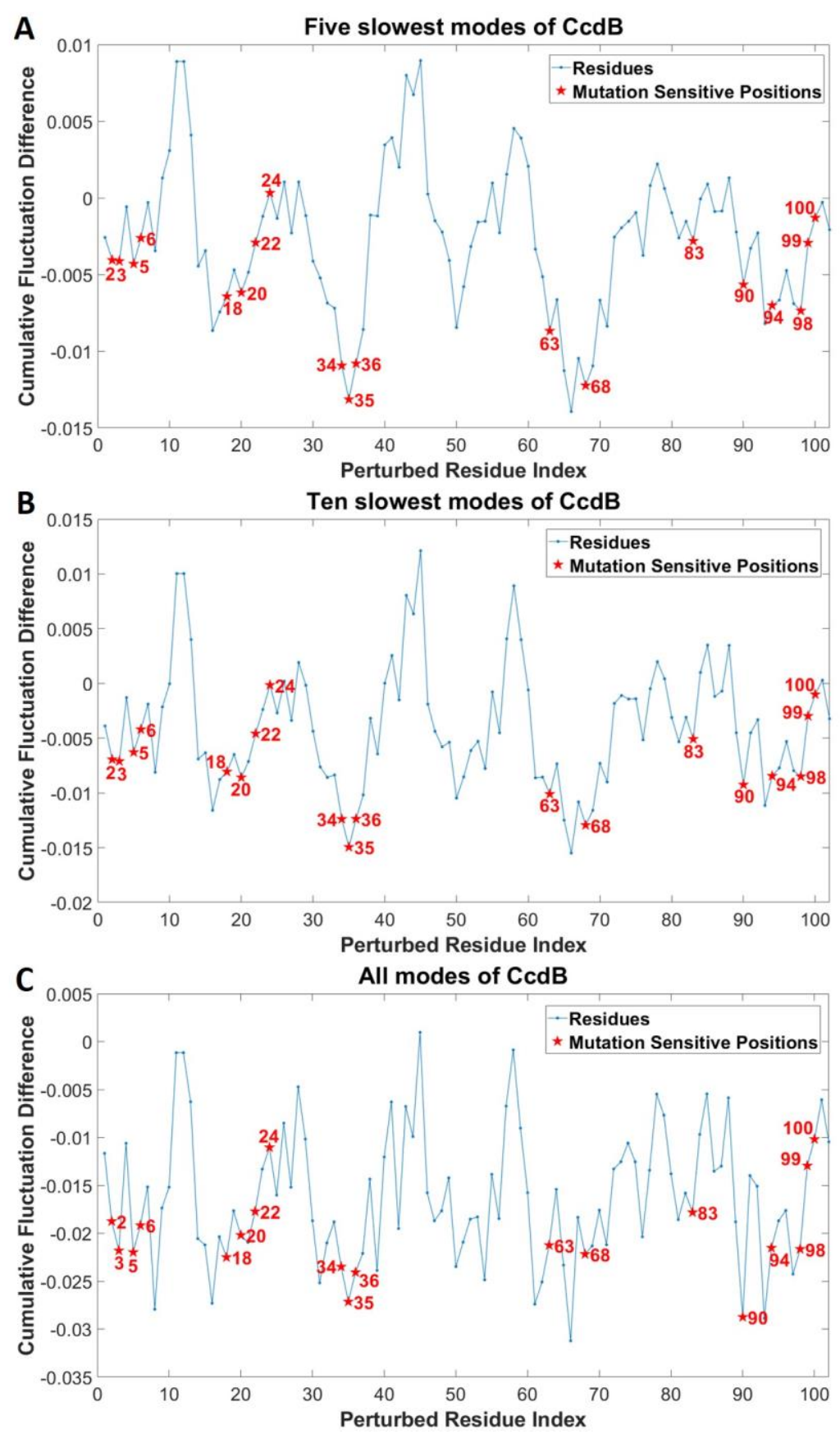

Figure S14. Cumulative residues fluctuations difference profiles of the $\mathrm{CcdB}$ domain upon straining perturbation on each residue for different mode selections. Mutation sensitive positions, detected using the deep sequencing data, are marked as red asterisks. (A) The five slowest modes. (B) The ten slowest modes. (C) All modes. 

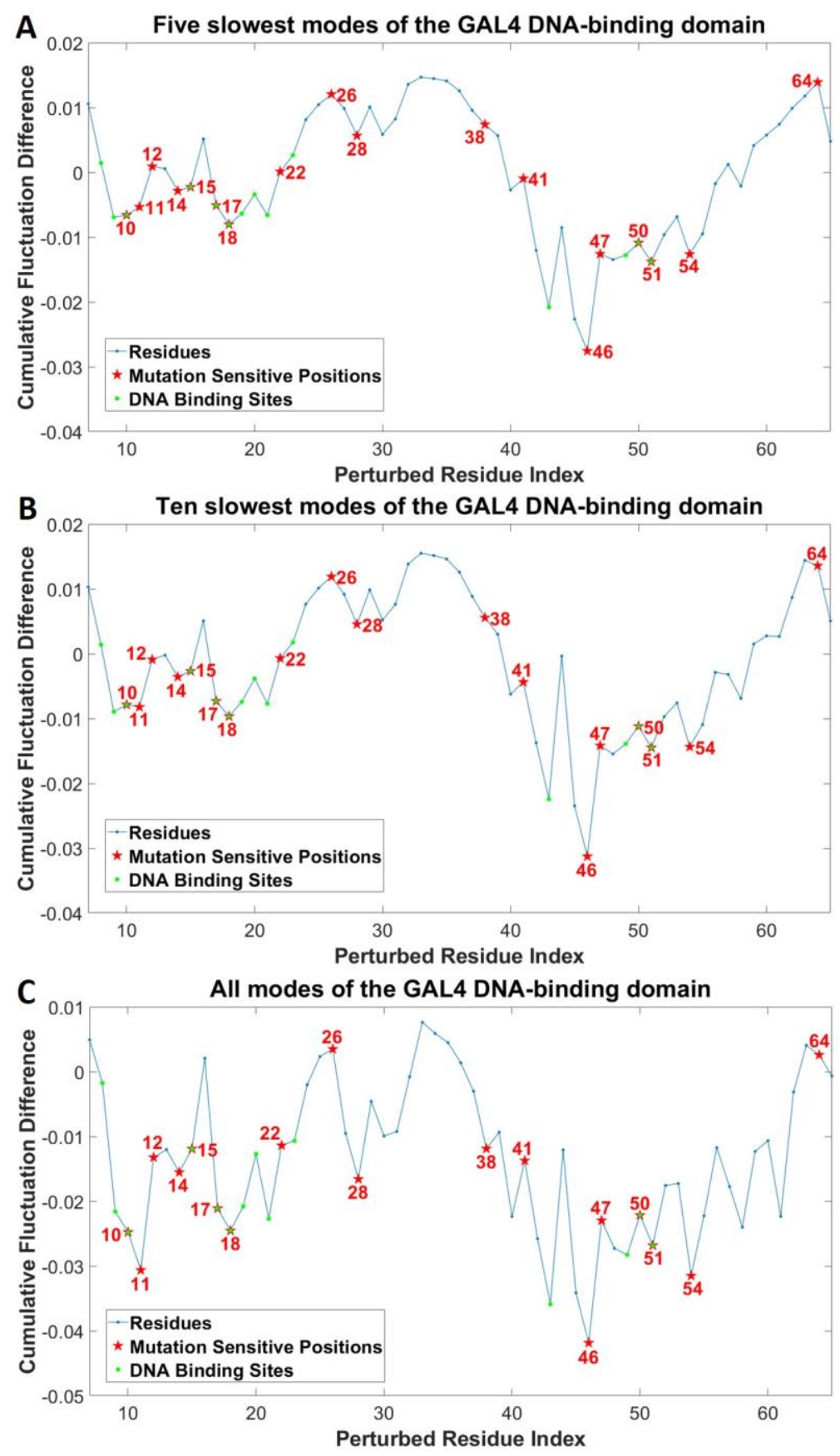

Figure S15. Cumulative residues fluctuations difference profiles of GAL4 DNA binding region upon straining perturbation on each residue for different mode selections. Mutation sensitive positions, detected using the deep sequencing data, are marked as red asterisks, and binding site residues as green asterisks. (A) The five slowest modes. (B) The ten slowest modes. (C) All modes. Binding site information is obtained from the crystal structure (PDB ID: 1D66) entry on PDBsum ${ }^{67}$. 

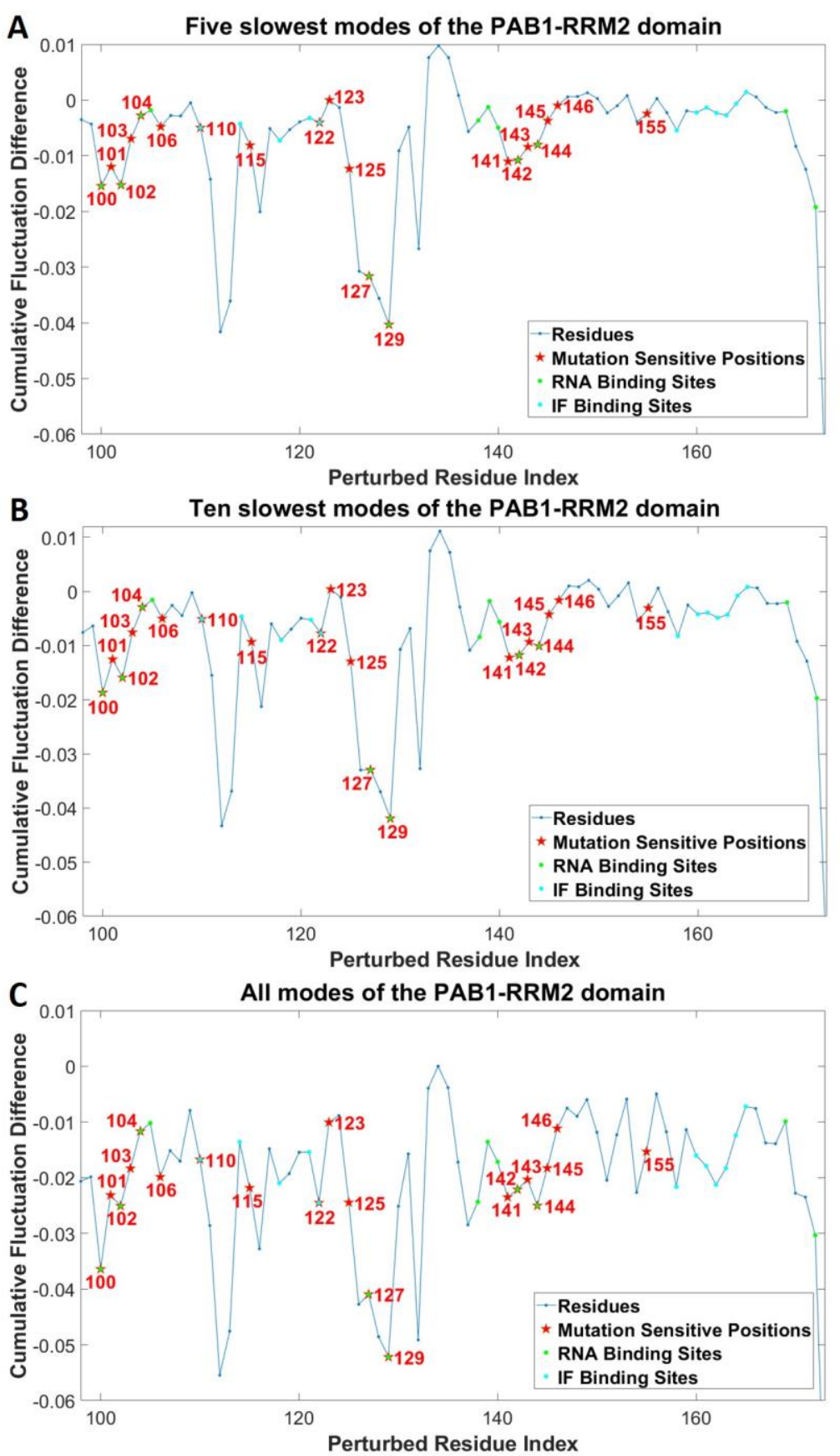

Figure S16. Cumulative residues fluctuations difference profiles of PAB1-RRM2 upon straining perturbation on each residue for different mode selections. Mutation sensitive positions, detected using the deep sequencing data, are marked as red asterisks, and binding site residues as green asterisks. (A) The five slowest modes. (B) The ten slowest modes (C) All modes. Binding site information is obtained from the crystal structure (PDB ID: 4F02) entry on PDBsum ${ }^{67}$. 

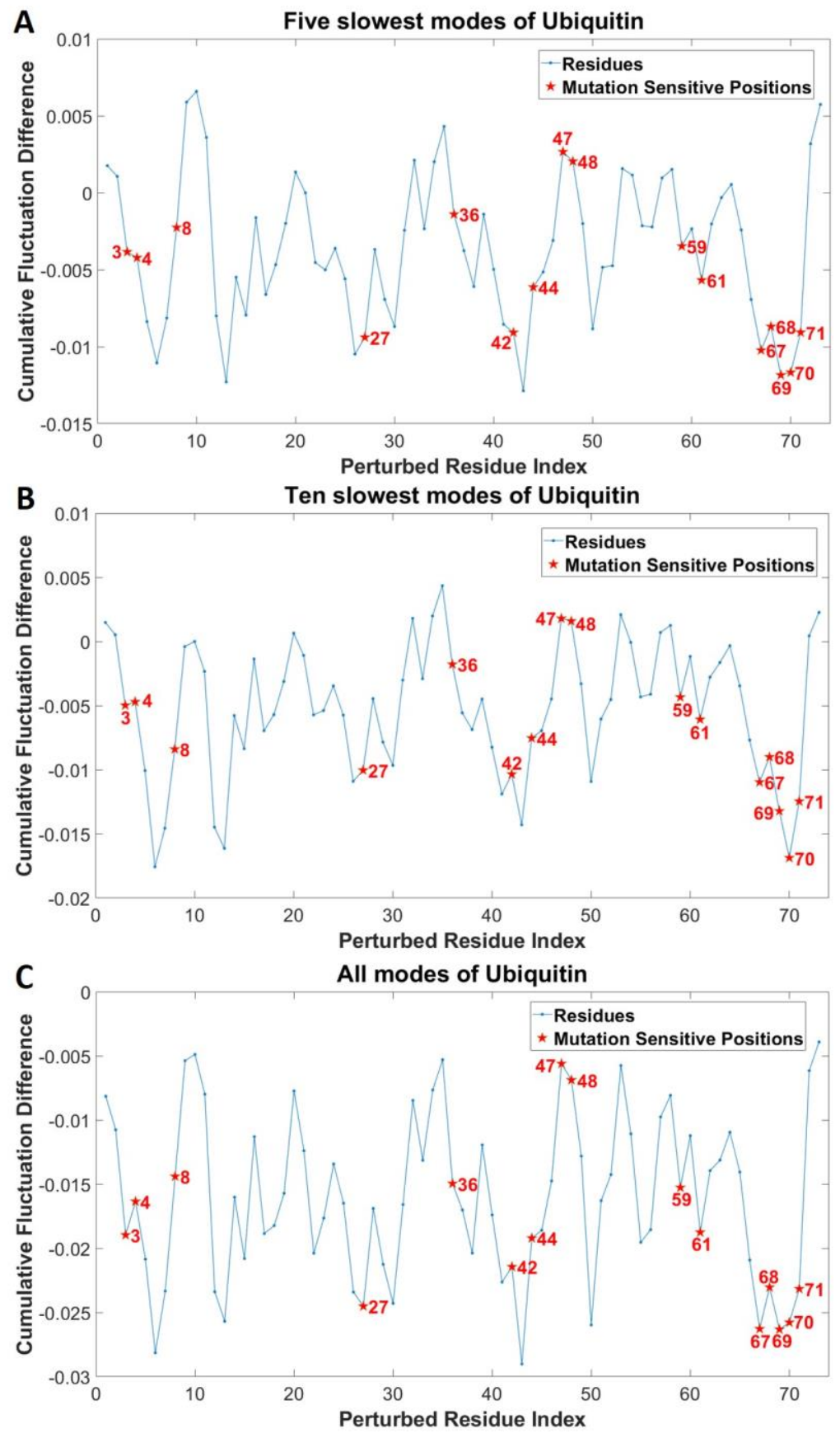

Figure S17. Cumulative residues fluctuations difference profiles of the ubiquitin domain upon straining perturbation on each residue for different mode selections. Mutation sensitive positions, detected using the deep sequencing data, are marked as red asterisks. (A) The five slowest modes. (B) The ten slowest modes. (C) All modes. 

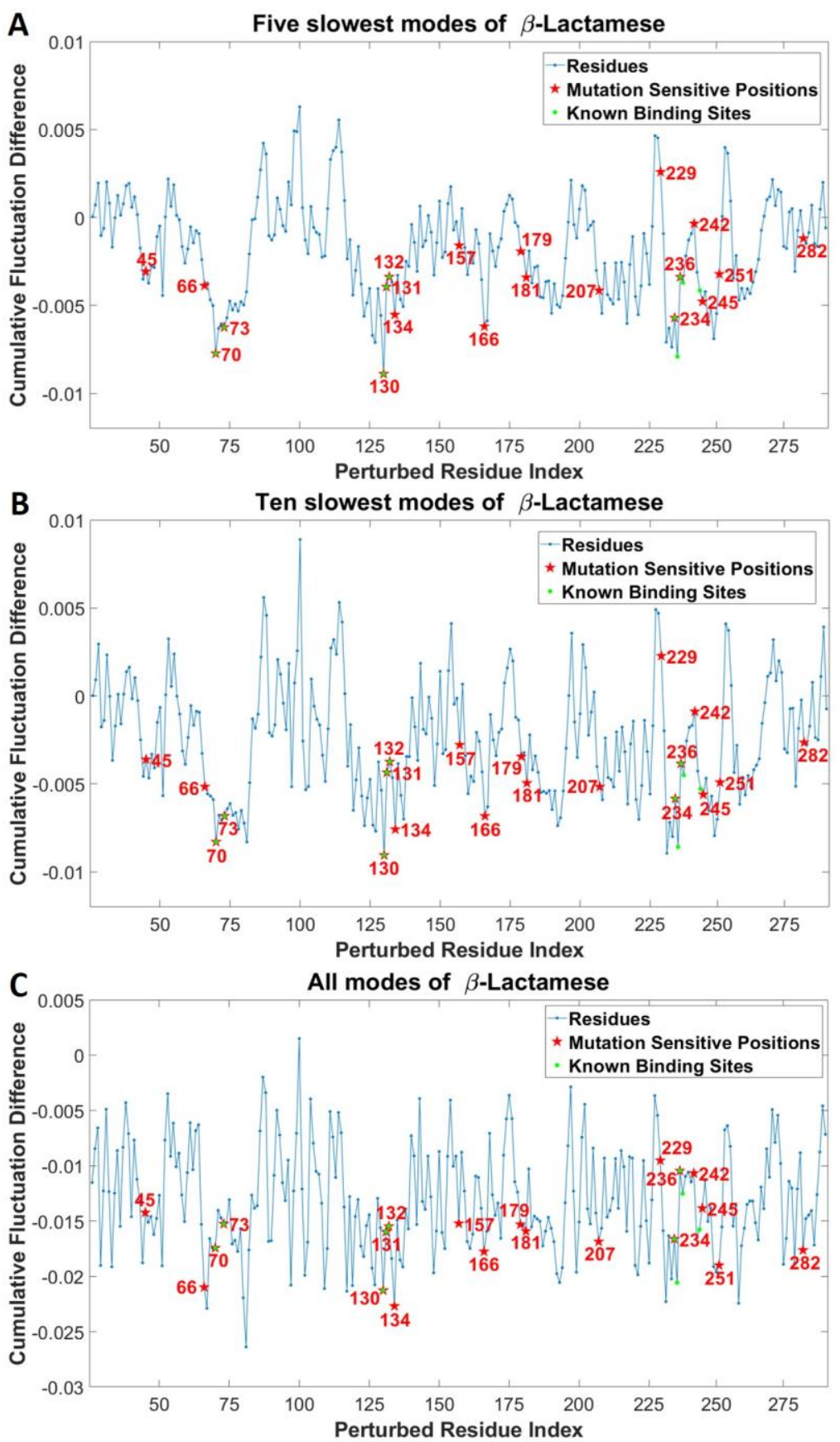

Figure S18. Cumulative residue fluctuations difference profiles of $\beta$-Lactamase upon straining perturbation on each residue for different mode selections. Mutation sensitive positions, detected using the deep sequencing data, are marked as red asterisks, and binding site residues as green asterisks. (A) The five slowest modes. (B) The ten slowest modes. (C) All modes. Binding site information is obtained from the crystal structure (PDB ID: 1XPB) entry on PDBsum ${ }^{67}$. 

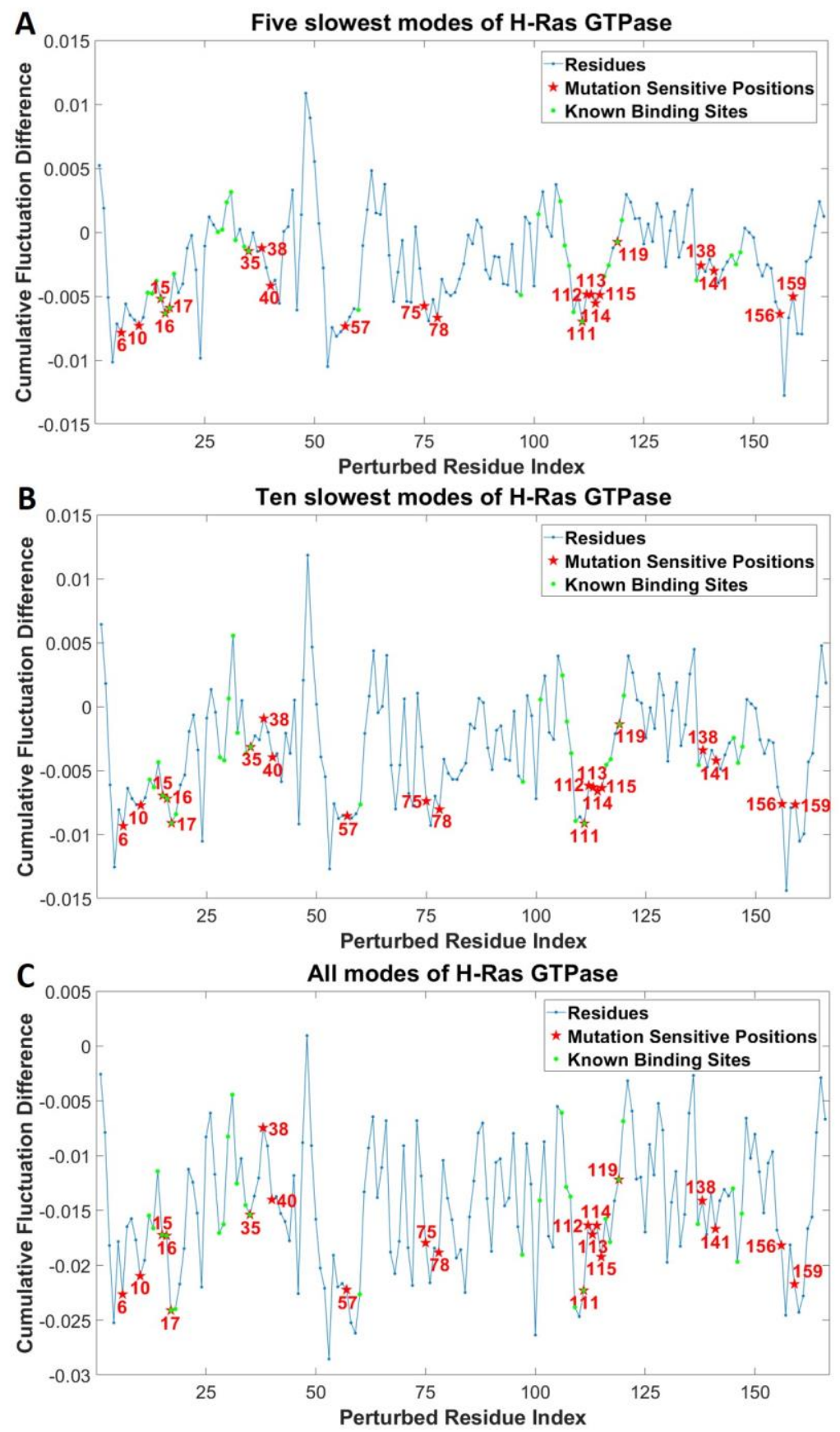

Figure S19. Cumulative residue fluctuations difference profiles of the H-Ras GTPase domain upon straining perturbation on each residue for different mode selections. Mutation sensitive positions, detected using the deep sequencing data, are marked as red asterisks, and binding site residues as green asterisks. (A) The five slowest modes. (B) The ten slowest modes. (C) All modes. Binding site information is obtained from the crystal structure (PDB ID: 3K8Y) entry on PDBsum ${ }^{67}$. 

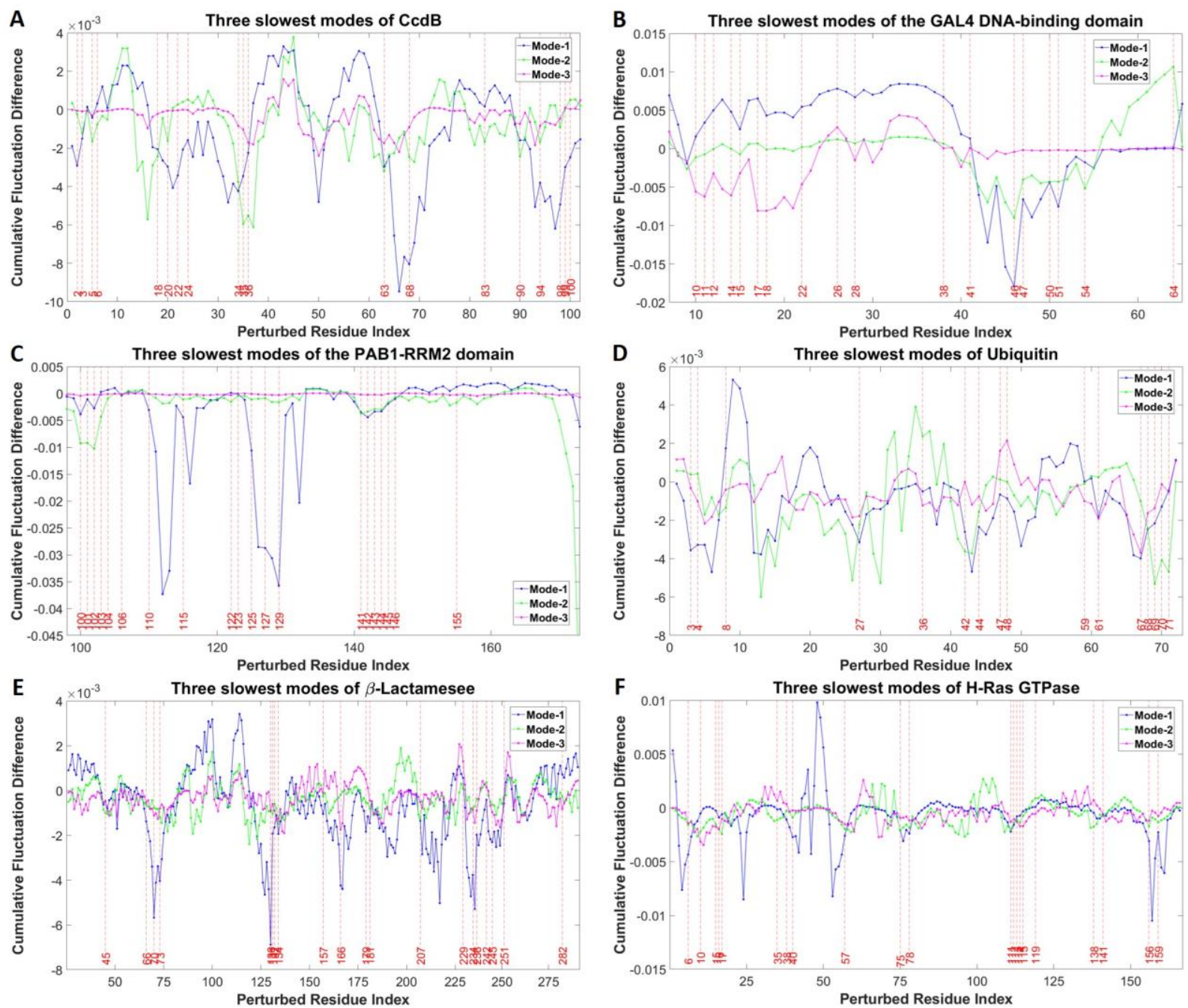

Figure S20. Cumulative residue fluctuation difference profile upon strain perturbation in the slowest, second slowest, and third slowest modes. Mutation sensitive positions, detected using the deep sequencing data, are marked in red dashed lines. (A) CcdB. (B) GAL4 DNA binding region. (C) PAB1-RRM2 Domain. (D) Ubiquitin. (E) TEM1 $\beta$-lactamase. (F) H-Ras GTPase. 

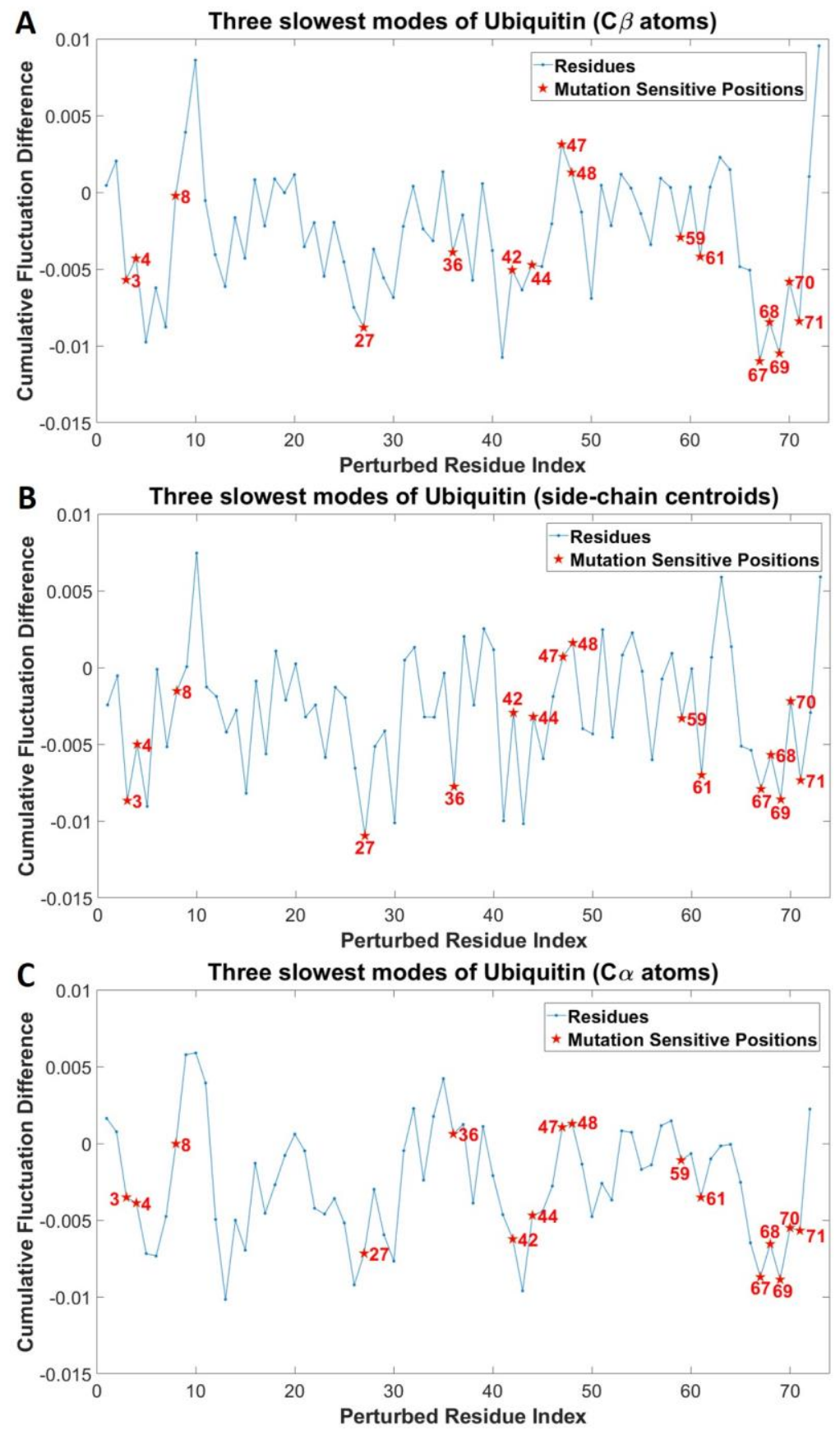

Figure S21. Cumulative residue fluctuation difference profiles of ubiquitin, calculated using higher resolution GNM models that take into account the $C \beta$ atoms (A), and side-chain centroids (B). (C) The results of the normal $\mathrm{C} \alpha$-based model for comparison. Mutation sensitive positions, detected using the deep sequencing data, are marked as red asterisks. 
Table S1. Statistical significance of the correlation between the mutation sensitive positions, detected in the deep sequencing experiments, and the dynamics perturbation analysis conducted here. Results are presented for the seven structures in our dataset, using different mode combinations (five slowest modes, ten slowest modes, and all modes). Statistical significance is reported for the number of mutation sensitive positions that overlap with the minima in the cumulative residue fluctuations profiles, as well as the distance between the mutation sensitive positions and the minima.

\begin{tabular}{|c|c|c|c|c|c|c|c|}
\hline Structure & Modes & $\begin{array}{l}\text { Number of mutation } \\
\text { sensitive positions } \\
\text { overlapping with } \\
\text { local minima }\end{array}$ & $\begin{array}{l}\text { Mean number of } \\
\text { random positions } \\
\text { overlapping with } \\
\text { local minima } \\
\text { (random sampling) }\end{array}$ & $p$-value & $\begin{array}{l}\text { Mean distance of } \\
\text { mutation sensitive } \\
\text { positions to local } \\
\text { minima }\end{array}$ & $\begin{array}{l}\text { Mean distance of } \\
\text { randomly selected } \\
\text { positions to local } \\
\text { minima }\end{array}$ & p-value \\
\hline \multirow{3}{*}{$\begin{array}{l}\text { PSD95-PDZ } \\
\text { Domain }\end{array}$} & 5 & 12 & 4.58 & $3.21 \times 10^{-6}$ & $0.94 \AA$ & $2.07 \AA$ & $6.89 \times 10^{-4}$ \\
\hline & 10 & 12 & 4.80 & $1.09 \times 10^{-5}$ & $0.64 \AA$ & $1.96 \AA$ & $6.58 \times 10^{-5}$ \\
\hline & All & 13 & 4.86 & $6.22 \times 10^{-7}$ & $0.70 \AA$ & $1.97 \AA$ & $8.21 \times 10^{-5}$ \\
\hline \multirow{3}{*}{ CcdB } & 5 & 8 & 2.81 & 0.0001 & $2.38 \AA$ & $3.50 \AA$ & 0.0290 \\
\hline & 10 & 9 & 4.53 & 0.0039 & $1.46 \AA ̊$ & $2.11 \AA ̊$ & 0.1192 \\
\hline & All & 7 & 4.51 & 0.0677 & $1.51 \AA$ & $1.88 \AA ̊$ & 0.2162 \\
\hline \multirow{3}{*}{$\begin{array}{l}\text { GAL4 - DNA } \\
\text { binding region }\end{array}$} & 5 & 5 & 3.47 & 0.1360 & $1.89 \AA$ & $2.94 \AA$ & 0.0398 \\
\hline & 10 & 5 & 3.16 & 0.0874 & $2.44 \AA$ & $3.55 \AA$ & 0.0507 \\
\hline & All & 4 & 3.15 & 0.2633 & $2.02 \AA$ & $3.02 \AA$ & 0.0442 \\
\hline \multirow{3}{*}{$\begin{array}{l}\text { PAB1-RRM2 } \\
\text { domain }\end{array}$} & 5 & 4 & 1.87 & 0.0289 & $3.15 \AA$ & $4.94 \AA ̊$ & 0.0046 \\
\hline & 10 & 5 & 2.92 & 0.0637 & $2.05 \AA$ & $3.66 \AA$ & 0.0025 \\
\hline & All & 6 & 4.00 & 0.0984 & $1.40 \AA ̊$ & $2.13 \AA$ & 0.0123 \\
\hline \multirow{3}{*}{ Ubiquitin } & 5 & 3 & 4.58 & 0.4260 & $2.35 \AA$ & $3.00 \AA ̊$ & 0.1621 \\
\hline & 10 & 4 & 2.95 & 0.2235 & $2.07 \AA$ & $2.82 \AA$ & 0.1164 \\
\hline & All & 5 & 3.16 & 0.0971 & $1.98 \AA ̊$ & $2.63 \AA$ & 0.1545 \\
\hline \multirow{3}{*}{$\begin{array}{c}\text { TEM1 } \\
\beta \text {-lactamase }\end{array}$} & 5 & 8 & 3.94 & 0.0084 & $1.64 \AA ̊$ & $2.63 \AA ̊$ & 0.0240 \\
\hline & 10 & 8 & 4.50 & 0.0256 & $1.48 \AA ̊$ & $2.19 \AA ̊$ & 0.0493 \\
\hline & All & 8 & 4.83 & 0.0412 & $1.17 \AA$ & $1.71 \AA$ & 0.0403 \\
\hline \multirow{3}{*}{ GTPase H-Ras } & 5 & 7 & 4.15 & 0.0491 & $1.55 \AA$ & $2.42 \AA$ & 0.0283 \\
\hline & 10 & 7 & 4.43 & 0.0726 & $1.28 \AA$ & $2.15 \AA$ & 0.0146 \\
\hline & All & 8 & 4.69 & 0.0336 & $1.23 \AA$ & $1.85 \AA$ & 0.0181 \\
\hline
\end{tabular}


Table S2. $p$-values obtained with different number of positions selected as mutation sensitive for the PSD95-PDZ domain. Different tiers of mutation sensitive positions are included in the selections. p-value analysis is conducted based on the distances between the mutation sensitive positions and the minima of cumulative residue fluctuations profiles calculated using the three-, five-, ten-, and all-slowest modes of motion.

\begin{tabular}{c|c|c|c|c|c}
\hline $\begin{array}{c}\text { Number of } \\
\text { Slow Modes }\end{array}$ & $\mathbf{1 2}$ & $\mathbf{1 7}$ & $\mathbf{2 0}$ & $\mathbf{2 7}$ & $\mathbf{3 6}$ \\
\hline $\mathbf{3}$ & 0.0190 & 0.0075 & 0.0012 & 0.0109 & 0.0017 \\
\hline $\mathbf{5}$ & 0.0032 & 0.0015 & $6.89 \times 10^{-4}$ & 0.0030 & $5.32 \times 10^{-4}$ \\
\hline $\mathbf{1 0}$ & $2.76 \times 10^{-4}$ & $1.17 \times 10^{-4}$ & $6.58 \times 10^{-5}$ & $1.25 \times 10^{-4}$ & $2.16 \times 10^{-5}$ \\
\hline All & $2.39 \times 10^{-4}$ & $1.91 \times 10^{-4}$ & $8.21 \times 10^{-5}$ & $3.81 \times 10^{-4}$ & $6.35 \times 10^{-5}$ \\
\hline
\end{tabular}


Table S3. p-values obtained with different number of positions selected as mutation sensitive for the deep sequencing dataset. $p$-value analysis is conducted based on the distances between the mutation sensitive positions and the minima of cumulative residue fluctuations profiles calculated using the three-, five-, ten-, and all-slowest modes of motion.

\begin{tabular}{|c|c|c|c|c|c|}
\hline Structure & $\begin{array}{c}\text { Number of } \\
\text { Positions Selected } \\
\text { as Mutation } \\
\text { Sensitive } \\
\end{array}$ & $\begin{array}{c}\text { p-value } \\
\text { (three slowest } \\
\text { modes) }\end{array}$ & $\begin{array}{c}\text { p-value } \\
\text { (five slowest } \\
\text { modes) }\end{array}$ & $\begin{array}{c}\text { p-value } \\
\text { (ten slowest } \\
\text { modes) }\end{array}$ & $\begin{array}{c}\text { p-value } \\
\text { (all modes) }\end{array}$ \\
\hline \multirow{3}{*}{$\mathrm{CcdB}$} & 11 & 0.1452 & 0.3794 & 0.3107 & 0.3308 \\
\hline & $\underline{19}$ & 0.0059 & 0.0290 & 0.1192 & 0.2162 \\
\hline & 32 & 0.0025 & 0.0218 & 0.0335 & 0.0769 \\
\hline \multirow{3}{*}{$\begin{array}{l}\text { GAL4 - DNA } \\
\text { binding } \\
\text { region }\end{array}$} & 11 & 0.0474 & 0.0953 & 0.1020 & 0.1110 \\
\hline & $\underline{18}$ & 0.0305 & 0.0398 & 0.0507 & 0.0442 \\
\hline & 32 & 0.0135 & 0.0061 & 0.0040 & 0.0003 \\
\hline \multirow{3}{*}{$\begin{array}{l}\text { PAB1-RRM2 } \\
\text { domain }\end{array}$} & 13 & 0.0026 & 0.0133 & 0.0051 & 0.0418 \\
\hline & $\underline{20}$ & $9.07 \times 10^{-4}$ & 0.0046 & 0.0025 & 0.0123 \\
\hline & 27 & 0.0001 & 0.0005 & 0.0002 & 0.0093 \\
\hline \multirow{3}{*}{ Ubiquitin } & 8 & 0.1036 & 0.3202 & 0.3807 & 0.4535 \\
\hline & $\underline{16}$ & 0.1730 & 0.1621 & 0.1164 & 0.1545 \\
\hline & 23 & 0.3550 & 0.5010 & 0.4863 & 0.4902 \\
\hline \multirow{3}{*}{$\begin{array}{c}\text { TEM1 } \\
\beta \text {-lactamase }\end{array}$} & 15 & 0.0130 & 0.0392 & 0.0823 & 0.1170 \\
\hline & $\underline{21}$ & 0.0071 & 0.0240 & 0.0493 & 0.0403 \\
\hline & 40 & $7.25 \times 10-5$ & $3.30 \times 10-5$ & $4.60 \times 10-4$ & 0.0215 \\
\hline \multirow{3}{*}{$\begin{array}{l}\text { H-Ras } \\
\text { GTPase }\end{array}$} & 11 & 0.0632 & 0.0926 & 0.0555 & 0.0894 \\
\hline & $\underline{21}$ & 0.0098 & 0.0283 & 0.0146 & 0.0181 \\
\hline & 29 & 0.0037 & 0.0066 & 0.0041 & 0.0050 \\
\hline
\end{tabular}


Table S4. $p$-values obtained with the GNM-based perturbation analysis based on the C $\beta$ atoms or side chain centroids instead of the Ca atoms. p-value analysis is conducted based on the distances between the mutation sensitive positions and the minima of cumulative residue fluctuations profiles calculated using the three slowest modes of motion. $p$-values obtained with the perturbation analysis based on the Ca atoms are given for ease of comparison.

\begin{tabular}{c|c|c|c} 
Structure & $\begin{array}{c}\mathbf{p} \text {-value } \\
\text { (C } \boldsymbol{\alpha} \text { atoms) }\end{array}$ & $\begin{array}{c}\mathbf{p} \text {-value } \\
\text { (C } \boldsymbol{\beta} \text { atoms) }\end{array}$ & $\begin{array}{c}\mathbf{p} \text {-value } \\
\text { (Side-chain centroids) }\end{array}$ \\
\hline $\begin{array}{c}\text { PSD95-PDZ } \\
\text { Domain }\end{array}$ & 0.0012 & 0.0221 & 0.0016 \\
\hline CcdB & 0.0059 & 0.0065 & 0.0111 \\
\hline $\begin{array}{c}\text { GAL4 - DNA } \\
\text { binding region }\end{array}$ & 0.0305 & 0.0493 & 0.0410 \\
\hline $\begin{array}{c}\text { PAB1-RRM2 } \\
\text { Domain }\end{array}$ & $9.07 \times 10-4$ & $6.35 \times 10^{-4}$ & $5.03 \times 10^{-4}$ \\
\hline $\begin{array}{c}\text { Ubiquitin } \\
\text { TEM1 }\end{array}$ & 0.1730 & 0.0285 & 0.0362 \\
\hline $\begin{array}{l}\text { P-lactamase } \\
\text { GTPase H-Ras }\end{array}$ & 0.0071 & 0.0131 & 0.0131 \\
\hline
\end{tabular}




\section{Supplementary Text}

\section{Mutation sensitive sites disclose intrinsic functional dynamic behavior}

Mapping mutation sensitive positions with their dynamic roles in the structure is of great value to decipher intrinsic structural and functional behavior.

CcdB functions by trapping the DNA gyrase and thus promoting cell death by increasing toxicity ${ }^{1}$. Survivability of cells is thus a measure of mutation sensitivity ${ }^{2}$, which is likely associated with the CcdB-DNA gyrase interaction or the stability of $\mathrm{CcdB}$ that affects this interaction. On the other hand, CcdB has an antitoxin partner CcdA that has opposing effect on toxicity ${ }^{3}$. Most of the minima of the cumulative residue fluctuations difference profile, V18-Q21, I34-L36, R48-L50, M63-T65 and 190/194, are mutation sensitive positions (save R48-L50). Interestingly, all are associated with the CcdA binding interaction sites. On the other hand, the minimum at L96/M97 and the highest maximum at 124-P28 are not mutation sensitive positions but rather the DNA gyrace binding sites. It is very interesting to observe the CcdA binding sites as the major player in the toxicity of $\mathrm{CcdB}$ in the deep sequencing experiments. CcdA binding sites likely modifies the DNA gyrace binding sites allosterically, although it does not exist either in the deep sequencing experiments or in the calculations. The observed behavior simply reflects an intrinsic dynamic behavior of $\mathrm{CcdB}$ in its interaction with $\mathrm{CcdA}$.

Since GAL4 is a transcription factor, the GAL4 data mostly reflects its binding to DNA. Many of the mutation sensitive positions are thus either DNA binding or metal binding residues ${ }^{4}$. The majority of mutation sensitive positions are located at one shallow minimum and one deep minimum of the cumulative residue fluctuation difference profile. On the other hand, R46 being in the vicinity of the DNA, although not a DNA binding site, has one of the highest functional costs and is located at the lowest minimum. As seen, binding sites may have dual dynamic behavior by displaying functional importance only by a direct interaction with the DNA and/or through their contribution to the global fluctuations of the structure. Of note, the dynamic response upon perturbation on GAL4 yields a better correlation with mutational sensitive positions when the DNA is included.

PAB1 interacts with poly-A tail of RNA and the initiation factor elF4G. The experimental data of $P A B 1$ focuses on its binding to initiation factor elF4G ${ }^{5}$. Many of the mutation sensitive positions of PAB1 are the RNA/initiation factor elF4G binding sites and tend to locate at two shallow minima of the cumulative residue fluctuations profile of the PAB1-elF4G complex. However, the mutational sensitive position $\mathrm{K} 129$ is not one of the elF4G binding sites but it is an RNA binding 
site at one of the deepest minima. N112 is not a mutational sensitive position but resides in another deep minimum in the immediate proximity of K129. K129 with its neighbor N112 must have a mechanistic importance in mediating allosteric interactions with the elF4G binding site, which is an intrinsic behavior in the dynamics of the PAB1-elF4G complex. Here as well, dual behavior of binding sites could be observed in their dynamic effect. The dynamic response of residues upon perturbation on PAB1-elF4G complex structure rather than only PAB1 structure results in a better correlation with mutation sensitive positions. If we perform analysis on PAB1 with or without RNA but without elF4G, the significance of the correlation worsens. Further, including RNA degrades the prediction. Maybe, in the order of events, elF4G binding precedes RNA binding.

In ubiquitin, including side chain centroids (Figure S16), improves the correlation between the minima of the cumulative residue fluctuations difference profile and the mutation sensitive positions of the fitness landscape of ubiquitin ${ }^{6}$. Except G47 and K48, mutation sensitive positions are either at the minima or right next to it in sequence. Special notes about functional importance of some residues: V26, K27, R42, Q49, L50, D52 ${ }^{7}$ were suggested to comprise a network of functional interactions; mutation of $I 30$ in contact with L15 and L43A significantly reduced the stability of ubiquitin ${ }^{8}$; I36 and Q41, which are hydrogen bonded to each other, were suggested to control slow dynamic conformational motions of ubiquitin ${ }^{9}$. All these residues could be identified here as leading to strong dynamic response upon perturbation.

From the fitness landscape of TEM1 $\boldsymbol{\beta}$-lactamase in the presence of ampicillin ${ }^{10}$, the lowest minima are at S70, K73, S130 and E166, the catalytic residues, are among the top mutation sensitive positions. Interesting to see the dynamic capacity of catalytic sites to modulate global fluctuations and possibly intermediate allosteric interactions. Residues E104 and M129) and residues L102, Y105, P107, K111, and M129 were identified as binding hotspots of two $\beta$ lactamase inhibitory proteins ${ }^{11}$. On the one hand, L190 was listed as a highly conserved residue at a minimum, although not a mutation sensitive position ${ }^{12}$. On the other hand, I263, T265, R275, Q278, I279 and L282 are not mutation sensitive positions but are at minima, which were shown to form a binding pocket for Xe when TEM-1 $\beta$-lactamase is used as a genetically encoded contrast agent for ${ }^{129}$ Xe hyper-CEST NMR ${ }^{13}$.

In the case of H-Ras GTPase, mutation sensitive positions reflect the sensitivity for GAP and GEF regulated Ras, leading to loss of Ras function ${ }^{14}$. Mutation sensitive positions are mainly at the minima of the cumulative residue fluctuations profile, where GNP (GTP analog) and ACT (acetate) binding sites could also be observed. On the other hand, L6, Y40, and F78 at the minima, although 
not binding residues, are mutational sensitive positions related with $\mathrm{P}$-loop and $\mathrm{Mg}+$ ion binding sites (nucleotide binding). Switch I (L23-K42) and Switch II (Q61-Y64, D69-R73, K101-K104) associated respectively with GAP and GEF binding are mostly away from the minima towards the maxima, however the core region (L52-G60, G75-F82, A155-V160) include mutation sensitive positions at the minima. Worth to note, the GNM based perturbation calculations are based on the crystal structure of Ras without GEF and GAP.

Finally, the GNM perturbations analysis could disclose the sites affecting global fluctuations that are mostly intrinsic in a given structure. However, considering the protein structure in different complex states would enhance the understanding of the functional dynamics. 


\section{References}

(1) Bernard, P.; Kézdy, K. E.; Van Melderen, L.; Steyaert, J.; Wyns, L.; Pato, M. L.; Higgins, P. N.; Couturier, M. The F Plasmid CcdB Protein Induces Efficient ATP-Dependent DNA Cleavage by Gyrase. J. Mol. Biol. 1993, 234 (3), 534-541.

(2) Adkar, B. V.; Tripathi, A.; Sahoo, A.; Bajaj, K.; Goswami, D.; Chakrabarti, P.; Swarnkar, M. K.; Gokhale, R. S.; Varadarajan, R. Protein Model Discrimination Using Mutational Sensitivity Derived from Deep Sequencing. Structure 2012, 20 (2), 371-381.

(3) Aghera, N. K.; Prabha, J.; Tandon, H.; Chattopadhyay, G.; Vishwanath, S.; Srinivasan, N.; Varadarajan, R. Mechanism of CcdA-Mediated Rejuvenation of DNA Gyrase. Structure 2020, 28 (5), 562-572.e4.

(4) Kitzman, J. O.; Starita, L. M.; Lo, R. S.; Fields, S.; Shendure, J. Massively Parallel SingleAmino-Acid Mutagenesis. Nat. Methods 2015, 12 (3), 203-206, 4 p following 206.

(5) Melamed, D.; Young, D. L.; Gamble, C. E.; Miller, C. R.; Fields, S. Deep Mutational Scanning of an RRM Domain of the Saccharomyces Cerevisiae Poly(A)-Binding Protein. RNA 2013, 19 (11), 1537-1551.

(6) Mavor, D.; Barlow, K.; Thompson, S.; Barad, B. A.; Bonny, A. R.; Cario, C. L.; Gaskins, G.; Liu, Z.; Deming, L.; Axen, S. D., et al. Determination of Ubiquitin Fitness Landscapes under Different Chemical Stresses in a Classroom Setting. Elife 2016, 5.

(7) Surana, P.; Das, R. Observing a Late Folding Intermediate of Ubiquitin at Atomic Resolution by NMR: Ubiquitin Intermediate at Atomic Resolution by NMR. Protein Sci. 2016, 25 (8), 1438-1450.

(8) Negi, H.; Reddy, P. P.; Vengayil, V.; Patole, C.; Laxman, S.; Das, R. A Novel Polyubiquitin Chain Linkage Formed by Viral Ubiquitin Is Resistant to Host Deubiquitinating Enzymes. Biochem. J. 2020, 477 (12), 2193-2219.

(9) Kitazawa, S.; Yagi-Utsumi, M.; Kato, K.; Kitahara, R. Interactions Controlling the Slow Dynamic Conformational Motions of Ubiquitin. Molecules 2017, 22 (9). https://doi.org/10.3390/molecules22091414.

(10) Firnberg, E.; Labonte, J. W.; Gray, J. J.; Ostermeier, M. A Comprehensive, HighResolution Map of a Gene's Fitness Landscape. Mol. Biol. Evol. 2014, 31 (6), 1581-1592. 
(11) Fryszczyn, B. G.; Adamski, C. J.; Brown, N. G.; Rice, K.; Huang, W.; Palzkill, T. Role of $\beta-$ Lactamase Residues in a Common Interface for Binding the Structurally Unrelated Inhibitory Proteins BLIP and BLIP-II: Role of $\beta$-Lactamase Residues in a Common Interface. Protein Sci. 2014, 23 (9), 1235-1246.

(12) Philippon, A.; Slama, P.; Dény, P.; Labia, R. A Structure-Based Classification of Class A $\beta$-Lactamases, a Broadly Diverse Family of Enzymes. Clin. Microbiol. Rev. 2016, 29 (1), 29-57.

(13) Roose, B. W.; Zemerov, S. D.; Wang, Y.; Kasimova, M. A.; Carnevale, V.; Dmochowski, I. J. A Structural Basis for 129 Xe Hyper-CEST Signal in TEM-1 $\beta$-Lactamase. Chemphyschem 2019, 20 (2), 260-267.

(14) Bandaru, P.; Shah, N. H.; Bhattacharyya, M.; Barton, J. P.; Kondo, Y.; Cofsky, J. C.; Gee, C. L.; Chakraborty, A. K.; Kortemme, T.; Ranganathan, R., et al. Deconstruction of the Ras Switching Cycle through Saturation Mutagenesis. Elife 2017, 6. 